\title{
MAXIMAL ORDERS OVER REGULAR LOCAL RINGS OF DIMENSION TWO
}

\author{
BY \\ MARK RAMRAS $\left({ }^{1}\right)$
}

Introduction. Auslander and Goldman [4] have studied maximal orders over discrete valuation rings. In this paper, relying heavily on their results, we investigate maximal orders over regular local rings of dimension two. The two theories are rather different. We begin $\$ 5$ by listing three theorems from [4]. Then we prove a partial generalization of two of them in dimension two (Theorem 5.4) and exhibit two examples which show that the remaining statements do not generalize.

In [4] a structure theorem is proved for maximal orders over discrete valuation rings which is the analogue of the Wedderburn structure theorem for simple artin rings. In $\S 6$ we extend this theorem, in a weakened form, to maximal orders over an arbitrary integrally closed noetherian domain $R$. We sharpen this somewhat when $R$ is regular local of dimension two and the maximal order is well behaved. Various other structure theorems are given in this section.

The first four sections are devoted to building up homological machinery, most of which is applied to orders in the last two sections. The setting is fairly general: $(R, \underline{m})$ is a commutative noetherian local ring with maximal ideal $\underline{m}$, and $\Lambda$ is an $R$-algebra which is finitely generated as an $R$-module. The best results (Theorems 1.10 and 2.16) are obtained when $\Lambda$ is quasi-local (i.e., $\Lambda / \operatorname{Rad} \Lambda$ is a simple artin ring, where $\operatorname{Rad} \Lambda$ is the Jacobson radical of $\Lambda$ ). Such rings behave very much like commutative local rings.

The author wishes to express his gratitude to Professor Maurice Auslander for his many helpful suggestions and his patient supervision of the research for this paper, which is the major portion of the author's doctoral dissertation. Many thanks also to Professor David Buchsbaum and Dr. Silvio Greco for useful and stimulating conversations.

Notations and conventions. Throughout this paper all rings have units and all modules are unitary. We use the abbreviations $\mathrm{pd}$, inj dim, and $\mathrm{gl}$ dim for projective, injective, and global dimension, respectively. Only when there is a possible leftright ambiguity will we write l.pd or r.inj dim, etc.

$R$ is a commutative noetherian ring and $\Lambda$ is an $R$-algebra which is finitely generated as an $R$-module. For the first four sections $R$ is local with maximal ideal $\underline{m}$. By $\hat{R}$ we will mean the completion of $R$ in the $\underline{m}$-adic topology, and if $M$ is an

Received by the editors June 11, 1968.

( $\left.{ }^{1}\right)$ Research on this paper was supported in part by US Army (Durham). 
$R$-module, $\hat{M}=M \otimes_{R} \hat{R}$. When our attention is focused on a particular element $y$ of $\underline{m}, \bar{M}$ will denote the module $M / y M$. Finally, $N=\operatorname{Rad} \Lambda$, the Jacobson radical of $\Lambda$.

1. Finistic global dimension. $\quad M$ is a finitely generated left $\Lambda$-module.

LEMMA 1.1. $\operatorname{pd}_{\Lambda} M=\operatorname{pd}_{\hat{\Lambda}} \hat{M}$.

Proof. Since $\hat{R}$ is $R$-flat, if $X$ is a $\Lambda$-projective resolution of $M$ then $X \otimes_{R}{ }^{\circ} \hat{R}$ is a $\hat{\Lambda}$-projective resolution of $\hat{M}$. Hence $\operatorname{pd}_{\hat{\Lambda}} \hat{M} \leqq \mathrm{pd}_{\Lambda} M$. On the other hand, suppose $\operatorname{pd}_{\Lambda} M \geqq n>0$. Then $\operatorname{Ext}_{\Lambda}^{n}(M, C) \neq 0$ for some finitely generated $\Lambda$-module $C$, by [8, Chapter VI, Proposition 2.5, p. 110]. Again, using the fact that $\hat{R}$ is $R$-flat we have $\operatorname{Ext}_{\Lambda}^{n}(M, C) \otimes_{R} \hat{R} \approx \operatorname{Ext}_{\hat{\Lambda}}^{n}(\hat{M}, \hat{C}) . \hat{R}$ is faithful on finitely generated $R$ modules, so $\operatorname{Ext}_{\hat{\Lambda}}^{n}(\hat{M}, \hat{C}) \neq 0$ and thus $\operatorname{pd}_{\hat{\Lambda}} \hat{M} \geqq n$. Therefore $\operatorname{pd}_{\hat{\Lambda}} \hat{M} \geqq \operatorname{pd}_{\Lambda} M$ and combining this with the first inequality we get the desired equality.

The next result is due to Silver [11].

LEMMA 1.2. For any integer $q \geqq 0$ the following are equivalent :

(a) $\operatorname{pd}_{\Lambda} M \leqq q$.

(b) $\operatorname{Ext}_{\Lambda}^{q+1}(M, \Lambda / N)=0$.

(c) $\operatorname{TOR}_{q+1}^{\Lambda}(\Lambda / N, M)=0$.

Corollary 1.3. $\mathrm{gl} \operatorname{dim} \Lambda=1 \cdot \mathrm{pd}_{\Lambda} \Lambda / N=\mathrm{r} \cdot \mathrm{pd}_{\Lambda} \Lambda / N$.

Proof. If $1 . \mathrm{pd}_{\Lambda} \Lambda / N=\infty$, then $1 . \mathrm{gl} \operatorname{dim} \Lambda=\infty$. Assume 1. $\mathrm{pd}_{\Lambda} \Lambda / N=k<\infty$. Then $\operatorname{TOR}_{k+1}^{\Lambda}(\Lambda / N,-)=0$. Thus by Lemma 1.2 if $A$ is any finitely generated left $\Lambda$-module, $\operatorname{pd}_{\Lambda} A \leqq k$. Thus sup $\left\{\operatorname{pd}_{\Lambda} A \mid A\right.$ a finitely generated left $\Lambda$-module $\}=k$. Hence by [1, Theorem 1] 1.gl $\operatorname{dim} \Lambda=1 \cdot \mathrm{pd}_{\Lambda} \Lambda / N$.

Similarly r.gl $\operatorname{dim} \Lambda=\mathrm{r} \cdot \mathrm{pd}_{\Lambda} \Lambda / N$. Finally, since $\Lambda$ is left and right noetherian, 1.gl $\operatorname{dim} \Lambda=\mathrm{r} . \mathrm{gl} \operatorname{dim} \Lambda$ [1, Corollary 5]. Thus $\mathrm{gl} \operatorname{dim} \Lambda=1 . \mathrm{pd}_{\Lambda} \Lambda / N=\mathrm{r} \cdot \mathrm{pd}_{\Lambda} \Lambda / N$.

Corollary 1.4. $\mathrm{gl} \operatorname{dim} \hat{\Lambda}=\mathrm{gl} \operatorname{dim} \Lambda$.

Proof. $\operatorname{Rad} \hat{\Lambda}=\hat{N}$, so $\hat{\Lambda} / \operatorname{Rad} \hat{\Lambda}=\hat{\Lambda} / \hat{N}=(\Lambda / N)^{\wedge}$. By Lemma $1.1 \operatorname{pd}_{\hat{\Lambda}}(\Lambda / N)^{\wedge}$ $=\operatorname{pd}_{\Lambda} \Lambda / N$. Applying Corollary 1.3, gl $\operatorname{dim} \hat{\Lambda}=\mathrm{gl} \operatorname{dim} \Lambda$.

Corollary 1.5. Suppose $\operatorname{pd}_{\Lambda} M=q<\infty$. Then there exists a simple left $\Lambda$-module $S$ and a simple right $\Lambda$-module $T$ such that $\operatorname{Ext}_{\Lambda}^{q}(M, S) \neq 0$ and $\operatorname{TOR}_{q}^{\Lambda}(T, M) \neq 0$.

Proof. $\Lambda / N$ is both left and right semisimple and is therefore a direct sum of simple left $\Lambda / N$-modules and also a direct sum of simple right $\Lambda / N$-modules. Simple $\Lambda / N$-modules and simple $\Lambda$-modules are the same. The corollary now follows from Lemma 1.2 and the fact that Ext and TOR commute with direct sums.

Definition. A noetherian ring $\Gamma$ is quasi-local if $\Gamma / \operatorname{Rad} \Gamma$ is a simple artin ring.

If $\Gamma$ is quasi-local then $\operatorname{Rad} \Gamma$ is the unique maximal two-sided ideal of $\Gamma$ and all simple left (right) $\Gamma$-modules are isomorphic.

Lemma 1.6. Suppose $\Lambda$ is quasi-local. 
(a) If $\operatorname{pd}_{\Lambda} M=q<\infty$ and $B$ is a right $\Lambda$-module which contains a simple right $\Lambda$-module $T$, then $\operatorname{TOR}_{q}^{\Lambda}(B, M) \neq 0$.

(b) If $B$ is a right $\Lambda$-module with $\operatorname{pd}_{\Lambda} B=k<\infty$ and $M$ contains a simple left $\Lambda$-module $S$, then $\operatorname{TOR}_{k}^{\Lambda}(B, M) \neq 0$.

Proof. (a) $0 \rightarrow T \rightarrow B \rightarrow B / T \rightarrow 0$ is exact. Thus so is

$$
\operatorname{TOR}_{q+1}^{\Lambda}(B / T, M) \rightarrow \operatorname{TOR}_{q}^{\Lambda}(T, M) \rightarrow \operatorname{TOR}_{q}^{\Lambda}(B, M) .
$$

The first term is 0 since $\operatorname{pd}_{\Lambda} M=q$. $\operatorname{TOR}_{q}^{\Lambda}(T, M) \neq 0$ by Corollary 1.5 and the fact that all simple right $\Lambda$-modules are isomorphic. Hence $\operatorname{TOR}_{q}^{\Lambda}(B, M) \neq 0$.

(b) The proof is nearly identical.

We recall some definitions from [3].

If $E$ is an $R$-module and $x \in \underline{m}, x$ is regular on $E$ if the homothety $E \stackrel{x}{\rightarrow} E$ is a monomorphism. A sequence $x_{1}, \ldots, x_{k}$ of elements of $\underline{m}$ is an E-sequence (or regular E-sequence) if $x_{1}$ is regular on $E$ and for $1<i \leqq k, x_{i}$ is regular on $E /\left(x_{1}, \ldots, x_{i-1}\right) E$ and $E /\left(x_{1}, \ldots, x_{k}\right) E \neq 0$. (When $E$ is finitely generated, this last condition is ensured by Nakayama's Lemma.) We denote by $\operatorname{codim}_{R} E$ the least upper bound of lengths of $E$-sequences. Since $R$ is noetherian, $\operatorname{codim}_{R} E$ is always finite.

LemMA 1.7. A finitely generated $\Lambda$-module $B$ contains a simple $\Lambda$-submodule $T$ if and only if $\operatorname{codim}_{R} B=0$.

Proof. $(\Rightarrow)$ Suppose $T \subset B, T$ a simple $\Lambda$-module. $\underline{m} T \varsubsetneqq T$ by Nakayama's Lemma, so $\underline{m} T=0$. Thus $\operatorname{codim}_{R} B=0$.

$(\Leftarrow)$ Assume $\operatorname{codim}_{R} B=0$. Then we have a monomorphism $R / \underline{m} \rightarrow B$. Let $C=\{b \in B \mid \underline{m} \cdot b=0\}=\operatorname{Hom}_{R}(R / \underline{m}, B)=\operatorname{Hom}_{\Lambda}(\Lambda / \underline{m} \Lambda, B) . C$ is a nonzero $\Lambda$ submodule of $B$ and $\underline{m} C=0$. Thus $C$ is a finite-dimensional vector space over the field $R / \underline{m}$ and therefore satisfies the descending chain condition on $R / \underline{m}$-submodules. But since every $\Lambda$-submodule of $C$ is also an $R / \underline{m}$-submodule, any descending chain of $\Lambda$-submodules of $C$ is finite. Therefore $C$ contains some simple $\Lambda$-submodule $T$ and we are done.

Lemma 1.8. Let $x_{1}, \ldots, x_{t}$ be a maximal $\Lambda$-sequence. Then

$$
1 . \mathrm{pd}_{\Lambda} \Lambda /\left(x_{1}, \ldots, x_{t}\right) \Lambda=\mathrm{r} \cdot \mathrm{pd}_{\Lambda} \Lambda /\left(x_{1}, \ldots, x_{t}\right) \Lambda=t,
$$

and $\operatorname{codim}_{R} \Lambda /\left(x_{1}, \ldots, x_{t}\right) \Lambda=0$.

Proof. Let $R_{x: 1, \ldots, t}$ be the Koszul complex on $x_{1}, \ldots, x_{t}$. Then $R_{x: 1, \ldots, t} \otimes_{R} \Lambda$ is acyclic and thus gives a free $\Lambda$-resolution of $\Lambda /\left(x_{1}, \ldots, x_{t}\right) \Lambda$ as both a left and a right $\Lambda$-module. The lemma now follows trivially.

The next lemma generalizes this result.

LemmA 1.9. Suppose $x \in \underline{m}$ and $x$ is regular on $M \neq 0$. Let $\bar{M}=M / x M$. Then $\operatorname{pd}_{\Lambda} \bar{M}=1+\operatorname{pd}_{\Lambda} M$. 
(If either one is infinite, so is the other.)

Proof. Tensoring the exact sequence $0 \rightarrow M \stackrel{x}{\rightarrow} M \rightarrow \bar{M} \rightarrow 0$ with $\Lambda / N$ gives us the long exact sequence

$$
\begin{aligned}
& \operatorname{TOR}_{q+1}^{\Lambda}(\Lambda / N, M) \longrightarrow \operatorname{TOR}_{q+1}^{\Lambda}(\Lambda / N, \bar{M}) \longrightarrow \operatorname{TOR}_{q}^{\Lambda}(\Lambda / N, M) \\
& \stackrel{x}{\longrightarrow} \operatorname{TOR}_{q}^{\Lambda}(\Lambda / N, M) .
\end{aligned}
$$

Claim: $\operatorname{TOR}_{q}^{\Lambda}(\Lambda / N, M)=0 \Leftrightarrow \operatorname{TOR}_{q+1}^{\Lambda}(\Lambda / N, \bar{M})=0$.

For suppose first that $\operatorname{TOR}_{q}^{\Lambda}(\Lambda / N, M)=0$. Then $\operatorname{pd}_{\Lambda} M \leqq q-1$ by Lemma 1.2. (Note: $q>0$ since by Nakayama's Lemma $\Lambda / N \otimes_{\Lambda} M \neq 0$.) Hence $\operatorname{TOR}_{q+1}^{\Lambda}(\Lambda / N, M)$ $=0$. We see from $\left(^{*}\right)$ that $\operatorname{TOR}_{q+1}^{\Lambda}(\Lambda / N, \bar{M})=0$.

Now suppose that $\operatorname{TOR}_{q+1}^{\Lambda}(\Lambda / N, \bar{M})=0$. Then

$$
0 \longrightarrow \operatorname{TOR}_{q}^{\Lambda}(\Lambda / N, M) \stackrel{x}{\longrightarrow} \operatorname{TOR}_{q}^{\Lambda}(\Lambda / N, M)
$$

is exact. But since $x \in \underline{m} \subset N, \Lambda / N \stackrel{x}{\rightarrow} \Lambda / N$ is the zero map. Therefore the induced map on $\operatorname{TOR}_{q}^{\Lambda}(\Lambda / N, M)$ is the zero map. Since it is also a monomorphism, $\operatorname{TOR}_{q}^{\Lambda}(\Lambda / N, M)=0$. This proves the claim. The lemma now follows directly from Lemima 1.2 .

THeOREM 1.10. Suppose $\Lambda$ is quasi-local. If $\mathrm{pd}_{\Lambda} M<\infty$, then

$$
\operatorname{pd}_{\Lambda} M+\operatorname{codim}_{R} M=\operatorname{codim}_{R} \Lambda \text {. }
$$

Proof. Let $y_{1}, \ldots, y_{d}$ be a maximal regular $M$-sequence and set

$$
M^{\prime}=M /\left(y_{1}, \ldots, y_{d}\right) M .
$$

Then by $d$ applications of Lemma 1.9 we have $\operatorname{pd}_{\Lambda} M+\operatorname{codim}_{R} M=\operatorname{pd}_{\Lambda} M^{\prime}$. So it suffices to prove that $\operatorname{pd}_{\Lambda} M^{\prime}=\operatorname{codim}_{R} \Lambda$.

Let $x_{1}, \ldots, x_{t}$ be a maximal $\Lambda$-sequence and let $\Lambda^{\prime}=\Lambda /\left(x_{1}, \ldots, x_{t}\right) \Lambda$. Then $\operatorname{codim}_{R} \Lambda^{\prime}=0=\operatorname{codim}_{R} M^{\prime}$. By Lemma $1.7 \Lambda^{\prime}$ contains a simple right $\Lambda$-module and $M^{\prime}$ contains a simple left $\Lambda$-module.

Let $\operatorname{pd}_{\Lambda} M^{\prime}=q$. According to Lemma 1.6 (a) $\operatorname{TOR}_{q}^{\Lambda}\left(\Lambda^{\prime}, M^{\prime}\right) \neq 0 . \operatorname{But} \mathrm{r} \cdot \mathrm{pd}_{\Lambda} \Lambda^{\prime}=t$ by Lemma 1.8 and so $q \leqq t$. On the other hand, by Lemma 1.6 (b) $\operatorname{TOR}_{t}^{\Lambda}\left(\Lambda^{\prime}, M^{\prime}\right)$ $\neq 0$. Since $\operatorname{pd}_{\Lambda} M^{\prime}=q, t \leqq q$. Therefore $q=t$, i.e., $\operatorname{pd}_{\Lambda} M^{\prime}=\operatorname{codim}_{R} \Lambda$.

LEMma 1.11. Suppose $\Lambda$ is quasi-local. Let $C(\Lambda)$ be the center of $\Lambda$. Then $C(\Lambda)$ is local, and for any finitely generated $\Lambda$-module $M, \operatorname{codim}_{R} M=\operatorname{codim}_{C(\Lambda)} M$.

Proof. $\Lambda \supset C(\Lambda) \supset R$ and $N \cap C(\Lambda)=\operatorname{Rad} C(\Lambda) \supset \underline{m}$. So $\Lambda / N \supset C(\Lambda) / \operatorname{Rad} C(\Lambda)$ $\supset R / \underline{m}$. Since $\Lambda / N$ is a simple artin ring its center is a field $F$. Clearly $F \supset$ $C(\Lambda) / \operatorname{Rad} C(\Lambda)$, so the latter is a domain. But it is a finite extension of the field $R / \underline{m}$ and is therefore itself a field. Thus $\operatorname{Rad} C(\Lambda)$ is the unique maximal ideal of $C(\Lambda)$, i.e., $\Lambda$ is local. 
Now let $y_{1}, \ldots, y_{d} \in \underline{m}$ be a maximal $M$-sequence. Since $\underline{m} \subset \operatorname{Rad} C(\Lambda)$ this is also an $M$-sequence from $C(\Lambda)$. Let $M^{\prime}=M /\left(y_{1}, \ldots, y_{d}\right) M \cdot \operatorname{Codim}_{C(\Lambda)} M=d$ $+\operatorname{codim}_{C(\Lambda)} M^{\prime}$.

By Lemma 1.7 , since $\operatorname{codim}_{R} M^{\prime}=0, M^{\prime}$ contains a simple $\Lambda$-module. Hence $\operatorname{codim}_{C(\Lambda)} M^{\prime}=0$ by the other half of Lemma 1.7. Thus $\operatorname{codim}_{C(\Lambda)} M=d=\operatorname{codim}_{R} M$.

Thus if $\Lambda$ is quasi-local, $\operatorname{codim}_{R} M$ (and in particular $\operatorname{codim}_{R} \Lambda$ ) is an invariant of $\Lambda$.

Definition. The left finitistic global dimension of $\Lambda$

(l.fin $g l \operatorname{dim} \Lambda$ ) $=\sup \left\{\operatorname{pd}_{\Lambda} M \mid \operatorname{pd}_{\Lambda} M<\infty, M\right.$ a finitely generated left $\Lambda$-module $\}$.

The right finitistic global dimension of $\Lambda$ (r.fin $\mathrm{gl} \operatorname{dim} \Lambda$ ) is the obvious analogue.

We may now rephrase the main theorem of this section.

THEOREM 1.12. Suppose $\Lambda$ is quasi-local. Then

l.fin $g l \operatorname{dim} \Lambda=$ r.fin $g l \operatorname{dim} \Lambda=\operatorname{codim}_{R} \Lambda=\operatorname{codim}_{C(\Lambda)} \Lambda$.

Corollary 1.13. If $\Lambda$ is quasi-local and $R$-free, then

fin $\mathrm{gl} \operatorname{dim} \Lambda=$ fin $\mathrm{gl} \operatorname{dim} R=\operatorname{codim}_{R} R$.

Proof. By Theorem $1.12 \mathrm{fin} \mathrm{gl} \operatorname{dim} \Lambda=\operatorname{codim}_{R} \Lambda$, and fin $\mathrm{gl} \operatorname{dim} R=\operatorname{codim}_{R} R$. Since $\Lambda$ is $R$-free $\operatorname{codim}_{R} \Lambda=\operatorname{codim}_{R} R$ and we get the desired result.

2. Injective dimension. Let $x \in \underline{m}$, and for any $R$-module $A, \bar{A}=A / x A . M$ is a finitely generated left $\Lambda$-module. We assume that $x$ is regular on $\Lambda$ and on $M$.

Lemma 2.1. Let $D$ be any left $\bar{\Lambda}$-module. Then for all $j \geqq 0$, $\operatorname{Ext}_{\Lambda}^{j}(M, D) \approx$ $\operatorname{Ext}_{\bar{\Lambda}}^{j}(\bar{M}, D)$.

Proof. This is a special case of [8, Chapter VI, Proposition 4.13, p. 118].

Setting $D=\Lambda / N$ and using Lemma 1.2 we obtain

Corollary 2.2. $\operatorname{pd}_{\Lambda} M=\operatorname{pd}_{\bar{\Lambda}} \bar{M}$.

Lemma 2.3. Suppose $\operatorname{Ext}_{\Lambda}^{j+1}(M, C)=0$, where $C$ is a left $\Lambda$-module on which $x$ is regular. Then

$$
\operatorname{Ext}_{\Lambda}^{j}(M, C) \otimes_{R} \bar{R} \approx \operatorname{Ext}_{\bar{\Lambda}}^{j}(\bar{M}, \bar{C}) .
$$

Proof. Applying $\operatorname{Ext}_{\Lambda}(M,-)$ to the exact sequence

$$
0 \longrightarrow C \stackrel{x}{\longrightarrow} C \longrightarrow \bar{C} \longrightarrow 0,
$$

we get the exact sequence

$$
\operatorname{Ext}_{\Lambda}^{j}(M, C) \stackrel{x}{\longrightarrow} \operatorname{Ext}_{\Lambda}^{j}(M, C) \longrightarrow \operatorname{Ext}_{\Lambda}^{j}(M, \bar{C}) \longrightarrow \operatorname{Ext}_{\Lambda}^{j+1}(M, C) .
$$

But $\operatorname{Ext}_{\Lambda}^{j+1}(M, C)=0$, so $\operatorname{Ext}_{\Lambda}^{j}(M, C) \otimes_{R} \bar{R} \approx \operatorname{Ext}_{\Lambda}^{j}(M, \bar{C})$. Combining this with Lemma 2,1 we have the desired isomorphism. 
Definition. For any $\Lambda$-module $E$,

$$
\operatorname{Ext}_{\Lambda}-\operatorname{dim} E=\sup \left\{j \geqq 0 \mid \operatorname{Ext}_{\Lambda}^{j}(E, \Lambda) \neq 0\right\} .
$$

If $\operatorname{Ext}_{\Lambda}^{j}(E, \Lambda)=0$ for all $j \geqq 0$, let $\operatorname{Ext}_{\Lambda}-\operatorname{dim} E=-\infty$.

Corollary 2.4. $\operatorname{Ext}_{\Lambda}-\operatorname{dim} M=\operatorname{Ext}_{\bar{\Lambda}}-\operatorname{dim} \bar{M}$.

Proof. Suppose $j \geqq 0$ and assume first that $\operatorname{Ext}_{\Lambda}-\operatorname{dim} M \leqq j$. Then $\operatorname{Ext}_{\Lambda}^{j+1}(M, \Lambda)$ $=0$ for all $i \geqq 1$. By Lemma $2.3 \operatorname{Ext}_{\bar{\Lambda}}^{j+i}(\bar{M}, \bar{\Lambda})=0$ so that $\operatorname{Ext}_{\bar{\Lambda}}-\operatorname{dim} \bar{M} \leqq j$. Since $x \in \underline{m}$ and $\operatorname{Ext}_{\Lambda}^{j}(M, \Lambda)$ is a finitely generated $R$-module, Nakayama's Lemma and Lemma 2.3 show that $\operatorname{Ext}_{\bar{\Lambda}}^{j}(\bar{M}, \bar{\Lambda})=0$ if and only if $\operatorname{Ext}_{\Lambda}^{j}(M, \Lambda)=0$. Thus $\operatorname{Ext}_{\Lambda}-\operatorname{dim} M=\operatorname{Ext}_{\bar{\Lambda}}-\operatorname{dim} \bar{M}$, provided that $\operatorname{Ext}_{\Lambda}-\operatorname{dim} M<\infty$. To complete the proof it suffices to show that if $\operatorname{Ext}_{\bar{\Lambda}}-\operatorname{dim} \bar{M}<\infty$, then $\operatorname{Ext}_{\Lambda}-\operatorname{dim} M<\infty$.

Assume that $\operatorname{Ext}_{\bar{\Lambda}}-\operatorname{dim} \bar{M} \leqq k$, where $0 \leqq k<\infty$. Then for all $i \geqq 1, \operatorname{Ext}_{\bar{\Lambda}}^{k+i}(\bar{M}, \bar{\Lambda})$ $=0$. By Lemma $2.1 \operatorname{Ext}_{\Lambda}^{k+i}(M, \bar{\Lambda})=0$. Hence from the exact sequence

$$
0 \longrightarrow \Lambda \stackrel{x}{\longrightarrow} \Lambda \longrightarrow \bar{\Lambda} \longrightarrow 0
$$

we get the exactness of

$$
\operatorname{Ext}_{\Lambda}^{k+i}(M, \Lambda) \stackrel{x}{\longrightarrow} \operatorname{Ext}_{\Lambda}^{k+i}(M, \Lambda) \longrightarrow 0 .
$$

Nakayama's Lemma implies that $\operatorname{Ext}_{\Lambda}^{k+i}(M, \Lambda)=0$. Since this is true for all $i \geqq 1$, Ext $_{\Lambda}-\operatorname{dim} M \leqq k<\infty$ and we are done.

The next lemma, whose proof we omit, is due to Matlis [10].

LEMmA 2.5. Let $B$ be any left $\Lambda$-module. Suppose $k>0$ and for all left ideals $I$ in $\Lambda, \operatorname{Ext}_{\Lambda}^{k}(\Lambda / I, B)=0$. Then inj $\operatorname{dim}_{\Lambda} B<k$.

As an immediate consequence:

Corollary 2.6. (a) $1 . \operatorname{inj} \operatorname{dim}_{\Lambda} \Lambda=\sup \left\{\operatorname{Ext}_{\Lambda}-\operatorname{dim} \Lambda / I \mid\right.$ I a left ideal $\}$.

(b) If $\operatorname{linj}_{\operatorname{dim}} \Lambda=t>0$, then

$$
t=1+\sup \left\{\operatorname{Ext}_{\Lambda}-\operatorname{dim} I \mid I \text { a left ideal }\right\} .
$$

Lemma 2.5 says that inj $\operatorname{dim}_{\Lambda} B$ depends only on the vanishing of $\operatorname{Ext}_{\Lambda}(-, B)$ on cyclic left $\Lambda$-modules. However, if $B$ is finitely generated, we may restrict our attention to simple left modules.

Proposition 2.7 (Auslander). Let $B$ be any nonzero, finitely generated left $\Lambda$-module.

(a) Suppose $k>0$ and for all simple left $\Lambda$-modules $S$ and for all $j \geqq k, \operatorname{Ext}_{\Lambda}^{j}(S, B)$ $=0$. Then inj $\operatorname{dim}_{\Lambda} B<k$.

(b) If $B$ is injective then for some simple $\Lambda$-module $S, \operatorname{Hom}_{\Lambda}(S, B) \neq 0$.

(c) Thus in general,

inj $\operatorname{dim}_{\Lambda} B=\sup \left\{k \geqq 0 \mid \operatorname{Ext}_{\Lambda}^{k}(S, B) \neq 0\right.$ for some simple left module $\left.S\right\}$. 
Proof. (a) Suppose inj $\operatorname{dim}_{\Lambda} B \geqq k$. Then by Lemma 2.5 , for some left ideal $I$, $\operatorname{Ext}_{\Lambda}^{k}(\Lambda / I, B) \neq 0$. Let $J$ be a left ideal in $\Lambda$, maximal with respect to the property that $\operatorname{Ext}_{\Lambda}^{j}(\Lambda / J, B) \neq 0$ for some $j \geqq k$. By the hypothesis $\Lambda / J$ cannot be simple. Clearly $J \neq \Lambda$. Suppose $\operatorname{codim}_{R} \Lambda / J=0$. Then there exists a $J^{\prime} \supsetneqq J$ such that $J^{\prime} / J$ is simple (Lemma 1.7). From the exact sequence

$$
0 \rightarrow J^{\prime} / J \rightarrow \Lambda / J \rightarrow \Lambda / J^{\prime} \rightarrow 0
$$

it follows that

$$
\operatorname{Ext}_{\Lambda}^{j}\left(\Lambda / J^{\prime}, B\right) \rightarrow \operatorname{Ext}_{\Lambda}^{j}(\Lambda / J, B) \rightarrow 0
$$

is exact.

Therefore $\operatorname{Ext}_{\Lambda}^{\}}\left(\Lambda / J^{\prime}, B\right) \neq 0$, which contradicts the assumed maximality of $J$. Thus $\operatorname{codim}_{R} \Lambda / J>0$. Let $y \in \underline{m}$ be $\Lambda / J$-regular. Then we have the exact sequence

$$
0 \longrightarrow \Lambda / J \stackrel{y}{\longrightarrow} \Lambda / J \longrightarrow \Lambda /(J, \Lambda y) \longrightarrow 0
$$

from which we obtain the exact sequence

$$
\operatorname{Ext}_{\Lambda}^{j}(\Lambda / J, B) \stackrel{y}{\longrightarrow} \operatorname{Ext}_{\Lambda}^{j}(\Lambda / J, B) \longrightarrow \operatorname{Ext}_{\Lambda}^{j+1}(\Lambda /(J, \Lambda y), B) .
$$

Since $\operatorname{Ext}_{\Lambda}^{j}(\Lambda / J, B) \neq 0$ and is finitely generated over $R$ because $B$ is finitely generated over $\Lambda, \operatorname{Ext}_{\Lambda}^{j+1}(\Lambda /(J, \Lambda y), B) \neq 0$, by Nakayama's Lemma. But $(J, \Lambda y) \supsetneqq J$ and $j+1>k$, so again we have a contradiction. Thus inj $\operatorname{dim}_{\Lambda} B<k$.

(b) Suppose inj $\operatorname{dim}_{\Lambda} B=0$.

Let $F=\left\{\right.$ left ideals $\left.I \mid \operatorname{Hom}_{\Lambda}(\Lambda / I, B) \neq 0\right\}$. Since $B \neq 0, \operatorname{Hom}_{\Lambda}(\Lambda, B) \neq 0$, so $0 \in F$ and thus $F \neq \varnothing$. Again, since $\Lambda$ is noetherian there is a maximal element $J$ in $F$. The proof now is nearly identical to the proof of part (a).

(c) Follows from (a) if inj $\operatorname{dim}_{\Lambda} B>0$ and from (b) if inj $\operatorname{dim}_{\Lambda} B=0$.

Corollary 2.8. $1 . \operatorname{inj} \operatorname{dim}_{\Lambda} \Lambda=\sup \left\{\operatorname{Ext}_{\Lambda}-\operatorname{dim} S \mid S\right.$ a simple left $\Lambda$-module $\}$.

Lemma 2.9. Suppose $E$ is a finitely generated left $\Lambda$-module and $\operatorname{pd}_{\Lambda} E=q<\infty$. Then $\operatorname{Ext}_{\Lambda}-\operatorname{dim} E=\mathrm{pd}_{\Lambda} E$.

Proof. Clearly $\operatorname{Ext}_{\Lambda}-\operatorname{dim} E \leqq q$.

$$
\operatorname{Ext}_{\Lambda}^{q}(E, \Lambda) \rightarrow \operatorname{Ext}_{\Lambda}^{q}(E, \Lambda / N) \rightarrow \operatorname{Ext}_{\Lambda}^{q+1}(E, N)
$$

is exact. Since $\operatorname{pd}_{\Lambda} E=q$, $\operatorname{Ext}_{\Lambda}^{q+1}(E, N)=0$ and by Lemma 1.2, $\operatorname{Ext}_{\Lambda}^{q}(E, \Lambda / N) \neq 0$. Hence $\operatorname{Ext}_{\Lambda}^{q}(E, \Lambda) \neq 0$. So $\operatorname{Ext}_{\Lambda}-\operatorname{dim} E=q$.

THEOREM 2.10. 1.inj $\operatorname{dim}_{\Lambda} \Lambda=1+1 . i n j \operatorname{dim}_{\bar{\Lambda}} \bar{\Lambda}$.

Proof. It follows from Bass [6, Theorem 2.2] that if 1.inj $\operatorname{dim}_{\Lambda} \Lambda<\infty$ then 1.inj $\operatorname{dim}_{\Lambda} \Lambda \geqq 1+1 . i n j \operatorname{dim}_{\bar{\Lambda}} \bar{\Lambda}$. Thus it suffices to show that if $1 . i n j \operatorname{dim}_{\bar{\Lambda}} \bar{\Lambda}<\infty$, 
then $1 . i n j \operatorname{dim}_{\Lambda} \Lambda \leqq 1+1 . i n j \operatorname{dim}_{\bar{\Lambda}} \bar{\Lambda}$. So assume that $1 . i n j \operatorname{dim}_{\bar{\Lambda}} \bar{\Lambda}=k<\infty$. Let $I$ be any left ideal of $\Lambda$. Then since $x$ is regular on $\Lambda$ it is regular on $I$. By Corollary 2.4,

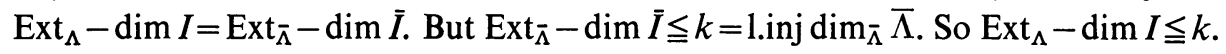
By Corollary 2.6, 1.inj $\operatorname{dim}_{\Lambda} \Lambda \leqq k+1$ and we are done.

Corollary 2.11. Suppose $\operatorname{codim}_{R} \Lambda=1$.inj $\operatorname{dim}_{\Lambda} \Lambda$. Then

$$
\text { r.inj } \operatorname{dim}_{\Lambda} \Lambda=1 . i n j \operatorname{dim}_{\Lambda} \Lambda \text {. }
$$

Proof. Let $\operatorname{codim}_{R} \Lambda=t$. Then there is a regular $\Lambda$-sequence $x_{1}, \ldots, x_{t}$, where $x_{i} \in \underline{m}$. Let $\Gamma=\Lambda /\left(x_{1}, \ldots, x_{t}\right) \Lambda$. By repeated application of Theorem 2.10 we see that $1 . i n j \operatorname{dim}_{\Gamma} \Gamma=0$. But then r.inj $\operatorname{dim}_{\Gamma} \Gamma=0$ by [9, Theorem 18]. The "right" analogue of Theorem 2.10 shows that $r . i n j \operatorname{dim}_{\Lambda} \Lambda=t$.

LEMMA 2.12. Let $E$ be a finitely generated left $\Lambda$-module, and assume that $y \in \underline{m}$ is regular on $E$. Then $\mathrm{Ext}_{\Lambda}-\operatorname{dim} E / y E=1+\mathrm{Ext}_{\Lambda}-\operatorname{dim} E$. (If $\mathrm{Ext}_{\Lambda}-\operatorname{dim} E= \pm \infty$, then $\operatorname{Ext}_{\Lambda}-\operatorname{dim} E / y E= \pm \infty$.)

Proof. Apply the functor $\operatorname{Ext}_{\Lambda}(-, \Lambda)$ to the exact sequence

$$
0 \longrightarrow E \stackrel{y}{\longrightarrow} E \longrightarrow E / y E \longrightarrow 0
$$

and use Nakayama's Lemma.

As an immediate consequence we have

CoROllary 2.13. $\operatorname{Ext}_{\Lambda}-\operatorname{dim} E+\operatorname{codim}_{R} E \leqq 1$.inj $\operatorname{dim}_{\Lambda} \Lambda$.

Proposition 2.14. Suppose $\Lambda$ is quasi-local and $1 . i n j \operatorname{dim}_{\Lambda} \Lambda=t<\infty$. If $E$ is any nonzero finitely generated left $\Lambda$-module then $\operatorname{Ext}_{\Lambda}-\operatorname{dim} E \geqq 0$ and $\mathrm{Ext}_{\Lambda}-\operatorname{dim} E$ $+\operatorname{codim}_{R} E=t$.

Proof. Let $\operatorname{codim}_{R} E=d$, and let $x_{1}, \ldots, x_{d}$ be a maximal regular $E$-sequence. Denote $E /\left(x_{1}, \ldots, x_{d}\right) E$ by $E^{\prime}$. Then $\operatorname{Ext}_{\Lambda}-\operatorname{dim} E+d=\operatorname{Ext}_{\Lambda}-\operatorname{dim} E^{\prime} \leqq t$. Since $\operatorname{codim}_{R} E^{\prime}=0, E^{\prime}$ contains a simple left $\Lambda$-module $S$. By Corollary 2.8 there exists a simple left $\Lambda$-module $S^{\prime}$ with $\operatorname{Ext}_{\Lambda}-\operatorname{dim} S^{\prime}=t$. But since $\Lambda$ is quasi-local, $S^{\prime}$ and $S$ are isomorphic, so that $\operatorname{Ext}_{\Lambda}-\operatorname{dim} S=t$. From the exact sequence

$$
0 \rightarrow S \rightarrow E^{\prime} \rightarrow E^{\prime} / S \rightarrow 0
$$

we obtain the exact sequence

$$
\operatorname{Ext}_{\Lambda}^{t}\left(E^{\prime}, \Lambda\right) \rightarrow \operatorname{Ext}_{\Lambda}^{t}(S, \Lambda) \rightarrow \operatorname{Ext}_{\Lambda}^{t+1}\left(E^{\prime} / S, \Lambda\right) .
$$

$\operatorname{Ext}_{\Lambda}^{t}(E, S) \neq 0$, and $\operatorname{Ext}_{\Lambda}^{t+1}\left(E^{\prime} / S, \Lambda\right)=0$ since 1.inj $\operatorname{dim}_{\Lambda} \Lambda=t$. Thus $\operatorname{Ext}_{\Lambda}^{t}\left(E^{\prime}, \Lambda\right)$ $\neq 0$. Hence $\operatorname{Ext}_{\Lambda}-\operatorname{dim} E^{\prime}=t$, and so $\operatorname{Ext}_{\Lambda}-\operatorname{dim} E+d=t$. This implies that Ext $_{\Lambda}-\operatorname{dim} E \geqq 0$ since $d<\infty$.

Corollary 2.15. If $\Lambda$ is quasi-local and $\operatorname{linj}^{\operatorname{dim}_{\Lambda}} \Lambda<\infty$ then $\operatorname{codim}_{R} \Lambda=$ 1.inj $\operatorname{dim}_{\Lambda} \Lambda$. Consequently

$$
\text { l.inj } \operatorname{dim}_{\Lambda} \Lambda=\text { fin } g l \operatorname{dim} \Lambda=\operatorname{rinj} \operatorname{dim}_{\Lambda} \Lambda \text {. }
$$


Proof. The first statement follows from Proposition 2.14 by setting $E=\Lambda$. The second comes from Theorem 1.12 and Corollary 2.11 .

We come now to the main theorem of this section.

THEOREM 2.16. Let $(R, \underline{m})$ be a regular local ring of dimension $n$. Suppose $\Lambda \supset R$ and $\Lambda$ is quasi-local. If $\operatorname{linj}_{\operatorname{dim}} \Lambda=t<\infty$ then $t=n$ and $\Lambda$ is $R$-free.

Proof. By induction on $t$. If $t=0$, then since $\Lambda$ is left and right noetherian $\Lambda$ satisfies the minimum condition on left ideals and on right ideals, by [9, Theorem 18]. Therefore $\operatorname{Rad} \Lambda$ is nilpotent. Since $\underline{m} \subset \operatorname{Rad} \Lambda$ and $R$ is a domain, $\underline{m}=(0)$. Thus $R$ is a field. Hence $n=0$ and $\Lambda$ is $R$-free.

Now suppose $t>0$ and assume the theorem is true for $t-1 . \operatorname{Codim}_{R} \Lambda=t \geqq 1$ by Corollary 2.15. Therefore $\underline{m} \notin \operatorname{Ass}_{R}(\Lambda)$ and it is well known that in fact there must be a $y \in \underline{m}-\underline{m}^{2}$ which is regular on $\Lambda$. Hence $1 . i n j \operatorname{dim}_{\Lambda / y \Lambda} \Lambda / y \Lambda=t-1$. Since $y \in \underline{m}-\underline{m}^{2}, R / y R$ is regular local of dimension $n-1$. Since $R$ is an integrally closed domain, $\Lambda \supset R$, and $\Lambda$ is finitely generated over $R$, it follows that $y \Lambda \cap R$ $=y R$. Hence $R / y R \subset \Lambda / y \Lambda$. Finally, $y \in \underline{m} \subset \operatorname{Rad} \Lambda$ and $\Lambda$ is quasi-local, so that $\Lambda / y \Lambda$ is quasi-local. Applying the induction hypothesis we get $n-1=t-1$ and $\Lambda / y \Lambda$ is $R / y R$-free. Therefore $n=t$ and $\Lambda$ is $R$-free.

If $\mathrm{gl} \operatorname{dim} \Lambda<\infty$, then $\mathrm{gl} \operatorname{dim} \Lambda=$ fin $g l \operatorname{dim} \Lambda=\operatorname{inj} \operatorname{dim}_{\Lambda} \Lambda$. Thus we have

COROLlaRY 2.17. Let $R$ be regular local of dimension $n$. Suppose $\Lambda$ is quasilocal and $\Lambda \supset R$. If $\mathrm{gl} \operatorname{dim} \Lambda<\infty$, then $\mathrm{gl} \operatorname{dim} \Lambda=n$ and $\Lambda$ is $R$-free.

3. The non-quasi-local case. We have seen (Theorem 2.16) that if $R$ is regular local, $\Lambda \supset R$ and $\Lambda$ is quasi-local, and linj $\operatorname{dim}_{\Lambda} \Lambda<\infty$, then $\operatorname{linj} \operatorname{dim}_{\Lambda} \Lambda=\mathrm{gl} \operatorname{dim} R$.

This statement is false if we remove the restriction that $\Lambda$ be quasi-local. For let $R$ be a field and let $\Lambda$ be the ring of $2 \times 2$ lower triangular matrices. It is well known that $\mathrm{gl} \operatorname{dim} \Lambda=1$. Consequently, $\operatorname{linj} \operatorname{dim}_{\Lambda} \Lambda=1$. On the other hand gl $\operatorname{dim} R=0$. Note that $\Lambda$ is not quasi-local since $\Lambda / N \simeq R \oplus R$ (ring direct sum).

Notice also that in this example fin $g l \operatorname{dim} \Lambda=1$ so that fin $g l \operatorname{dim} \Lambda>\operatorname{codim}_{R} \Lambda$ $=0$. So the condition that $\Lambda$ be quasi-local is also necessary in Theorem 1.12.

In this section we shall see what can be salvaged when we drop the assumption that $\Lambda$ is quasi-local.

Notation. If $S$ is a multiplicative subset of $R$ (i.e., $S \cdot S \subset S, 1 \in S$, and $0 \notin S$ ) and $E$ is an $R$-module, we will write $E_{S}$ for $E \otimes_{R} R_{S}$. If $S=R-p, p \in \operatorname{Spec} R$, we will write $E_{p}$ instead of $E_{R-p}$.

Proposition 3.1. Let $S$ be a multiplicative subset of $R$ such that every $s \in S$ is regular on $\Lambda$ (i.e., the natural map $\Lambda \rightarrow \Lambda_{S}$ is a mononorphism) and $\underline{m} \cap S \neq \varnothing$. Suppose 1.inj $\operatorname{dim}_{\Lambda} \Lambda=t<\infty$. Then l.inj $\operatorname{dim}_{\Lambda_{S}} \Lambda_{S}<1$.inj $\operatorname{dim}_{\Lambda} \Lambda$.

Proof. We first show that $1 . \operatorname{inj} \operatorname{dim}_{\Lambda_{S}} \Lambda_{S} \leqq t$. If $A$ is any cyclic left $\Lambda_{S}$-module, $A \simeq B_{S}$ for some cyclic left $\Lambda$-module $B$. Since $R_{S}$ is $R$-flat, $\operatorname{Ext}_{\Lambda_{S}}^{q}\left(B_{S}, \Lambda_{S}\right) \approx$ 
$\operatorname{Ext}_{\Lambda}^{q}(B, \Lambda) \otimes_{R} R_{S}$ by [4, Lemma 2.4]. Thus $\operatorname{Ext}_{\Lambda_{S}}^{q}\left(A, \Lambda_{S}\right)=0$ for all $q>t$ and all cyclic left $\Lambda_{S}$-modules $A$. Hence $1 . i n j \operatorname{dim}_{\Lambda_{S}} \Lambda_{S} \leqq t$ by Lemma 2.5 .

Now suppose linj $\operatorname{dim}_{\Lambda_{S}} \Lambda_{S}=t$. Then by Corollary 2.6 there is a left ideal $J$ in $\Lambda_{S}$ with $\operatorname{Ext}_{\Lambda_{S}}-\operatorname{dim} \Lambda_{S} / J=t$. Let $I=J \cap \Lambda . I$ is a left ideal in $\Lambda$ and $I_{S}=J$. Therefore $t \geqq \operatorname{Ext}_{\Lambda}-\operatorname{dim} \Lambda / I \geqq \operatorname{Ext}_{\Lambda_{S}}-\operatorname{dim}(\Lambda / I)_{S}=t$. So $\operatorname{Ext}_{\Lambda}-\operatorname{dim} \Lambda / I=t$. Hence $\operatorname{codim}_{R} \Lambda / I$ $=0$ (Corollary 2.13) and so $\underline{m} \in \operatorname{Ass}_{R}(\Lambda / I)$. Since $J \cap \Lambda=I, 0 \rightarrow \Lambda / I \rightarrow(\Lambda / I)_{S}$ is exact. $0 \rightarrow R / \underline{m} \rightarrow \Lambda / I$ is also exact and we get a commutative diagram:

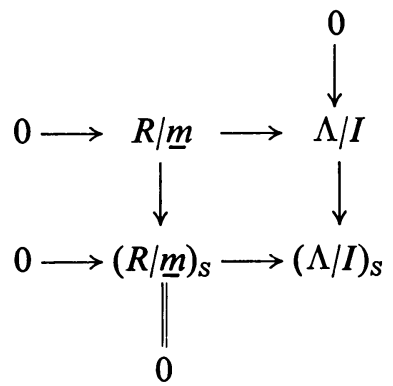

Since $\underline{m} \cap S \neq \varnothing, \underline{m}_{S}=R_{S}$, so $(R / \underline{m})_{S}=0$. The commutativity of the diagram says that the map $R / \underline{m} \rightarrow(\Lambda / I)_{S}$ is both a monomorphism and the zero map, which is absurd.

Therefore l.inj $\operatorname{dim}_{\Lambda_{S}} \Lambda_{S}<1$.inj $\operatorname{dim}_{\Lambda} \Lambda$.

CoRollaRy 3.2. Suppose $R$ has Krull dimension $n$ and $\Lambda$ is torsion-free over $R$. Then l.inj $\operatorname{dim}_{\Lambda} \Lambda \geqq n$.

Proof. By induction on $n$. There is nothing to prove when $n=0$, so assume that $n>0$ and the result is true for $n-1$. There is a $p \in \operatorname{Spec} R$ such that Krull $\operatorname{dim} R_{p}$ $=n-1$. $\Lambda_{p}$ is torsion-free over $R_{p}$ so by the induction hypothesis linj $\operatorname{dim}_{\Lambda_{p}} \Lambda_{p}$ $\geqq n-1$. $p \neq \underline{m}$, so $\underline{m} \cap R-p \neq \varnothing$. By the preceding proposition,

$$
\text { l.inj } \operatorname{dim}_{\Lambda_{p}} \Lambda_{p}<1 . \operatorname{inj} \operatorname{dim}_{\Lambda} \Lambda \text {. }
$$

Therefore l.inj $\operatorname{dim}_{\Lambda} \Lambda \geqq n$.

COROllary 3.3. Let $R$ be regular local of dimension $n$ and assume that $\Lambda$ is torsion-free over $R$. Let $p \in \operatorname{Spec} R$. Suppose $1 . i n j \operatorname{dim}_{\Lambda} \Lambda=n$.

Then $1 \operatorname{inj} \operatorname{dim}_{\Lambda_{p}} \Lambda_{p}=\mathrm{gl} \operatorname{dim} R_{p}$.

Proof. By induction on $n$. The case $n=0$ is trivial so assume $n>0$ and the result is true for $n-1$. If $p=\underline{m}$ there is nothing to prove, so assume that $p \varsubsetneqq \underline{m}$. There exists an $\mathscr{F} \in \operatorname{Spec} R$ such that $p \subset \mathscr{F} \varsubsetneqq \underline{m}$ with no prime of $R$ properly between $\mathscr{F}$ and $\underline{m}$. Thus gl $\operatorname{dim} R_{\mathscr{F}}=$ Krull $\operatorname{dim} R_{\mathscr{F}}=n-1$. By Proposition 3.1

$$
\text { l.inj } \operatorname{dim}_{\Lambda \mathscr{F}} \Lambda_{\mathscr{F}}<1 . i n j \operatorname{dim}_{\Lambda} \Lambda=n \text {. }
$$

Thus 1.inj $\operatorname{dim}_{\Lambda \mathscr{F}} \Lambda_{\mathscr{F}} \leqq n-1$. By Corollary 3.2 1.inj $\operatorname{dim}_{\Lambda_{\mathscr{F}}} \Lambda_{\mathscr{F}} \geqq n-1$, so l.inj $\operatorname{dim}_{\Lambda_{F}} \Lambda_{\mathscr{F}}=n-1$. Since $\Lambda_{p}=\left(\Lambda_{\mathscr{F}}\right)_{p}, R_{p}=\left(R_{\mathscr{F}}\right)_{p}$, and $\mathrm{gl} \operatorname{dim} R_{\mathscr{F}}=n-1$, the induction hypothesis gives $1 . i n j \operatorname{dim}_{\Lambda_{p}} \Lambda_{p}=\operatorname{gl} \operatorname{dim} R_{p}$. 
COROllaRy 3.4. Let $R$ be regular local of dimension $n$ and let $\Lambda$ be torsion-free over $R$. Suppose $\mathrm{gl} \operatorname{dim} \Lambda=n$. Then for any $p \in \operatorname{Spec} R, g l \operatorname{dim} \Lambda_{p}=\operatorname{gl} \operatorname{dim} R_{p}$. Consequently, if $S$ is any multiplicative subset of $R, \mathrm{gl} \operatorname{dim} \Lambda_{S}=\mathrm{gl} \operatorname{dim} R_{S}$.

Proof. The first statement follows from Corollary 3.3 and the fact that gl dim $\Gamma$ $=\operatorname{inj} \operatorname{dim}_{\Gamma} \Gamma$ when $g l \operatorname{dim} \Gamma<\infty$. For the second statement, if $\Gamma$ is an $R$-algebra and a left noetherian ring then

$$
\mathrm{gldim} \Gamma_{S}=\sup \left\{\mathrm{gl} \operatorname{dim} \Gamma_{p} \mid p \in \operatorname{Spec} R, p \cap S=\varnothing\right\} .
$$

We conclude this section with a simple criterion for $\Lambda$ and $R$ to have the same global dimension when $\Lambda$ is $R$-free.

Proposition 3.5. Suppose $R$ is regular local of dimension $n$ and $\Lambda$ is $R$-free. Then $\mathrm{gl} \operatorname{dim} \Lambda=n \Leftrightarrow$ every finitely generated $R$-free $\Lambda$-module is $\Lambda$-projective.

Proof. $(\Rightarrow)$ Let $E$ be a finitely generated $R$-free $\Lambda$-module. Then $\operatorname{codim}_{R} E$ $=\operatorname{codim}_{R} R=n$ so there is a regular $E$-sequence $x_{1}, \ldots, x_{n}$. By Lemma 1.9 we get $\operatorname{pd}_{\Lambda} E /\left(x_{1}, \ldots, x_{n}\right) E=n+\operatorname{pd}_{\Lambda} E$. But $\operatorname{pd}_{\Lambda} E /\left(x_{1}, \ldots, x_{n}\right) E \leqq \operatorname{gldim} \Lambda=n$, so $\operatorname{pd}_{\Lambda} E$ $=0$, i.e., $E$ is $\Lambda$-projective.

$(\Leftarrow) \operatorname{gl} \operatorname{dim} \Lambda=\sup \left\{\operatorname{pd}_{\Lambda} M \mid M\right.$ a finitely generated $\Lambda$-module $\}$. (In fact, by [1, Theorem 1] we may restrict $M$ to range over cyclic modules.)

Let $0 \rightarrow E \rightarrow P_{n-1} \rightarrow \cdots \rightarrow P_{0} \rightarrow M \rightarrow 0$ be exact with each $P_{i}$ a finitely generated $\Lambda$-projective module. Since $\Lambda$ is $R$-free, so is $P_{i}$. But $\operatorname{pd}_{R} M \leqq n=\operatorname{gldim} R$, so $E$ must be $R$-free. Therefore by hypothesis $E$ is $\Lambda$-projective. Thus $\operatorname{pd}_{\Lambda} M \leqq n$. Hence $\mathrm{gl} \operatorname{dim} \Lambda \leqq n$. But since $\Lambda$ is $R$-free, a regular $R$-sequence $x_{1}, \ldots, x_{n}$ is also a regular $\Lambda$-sequence, and so $\operatorname{pd}_{\Lambda} \Lambda /\left(x_{1}, \ldots, x_{n}\right) \Lambda=n$. Therefore $\mathrm{gl} \operatorname{dim} \Lambda=n$.

4. A theorem on global dimension. If $(R, \underline{m})$ is regular local of dimension $n$ and $x \in \underline{m}-\underline{m}^{2}$, it is a well-known fact that $\bar{R}$ is regular local of dimension $n-1$. In this section we show how the proof carries over to the noncommutative case. Here we shall assume that $\Lambda$ is local, i.e., that $\Lambda / N$ is a division ring. At the end of $\S 6$ we extend the theorem to quasi-local rings.

LEMMA 4.1. Let $(R, \underline{m})$ be any noetherian local ring. Suppose $\Lambda$ is local and $x \in \underline{m}-N^{2}$. Then $\Lambda / N$ is a $\bar{\Lambda}$-direct summand of $\bar{N}$.

Proof. Since $\Lambda / N$ is a division ring and $x \in N-N^{2},\left\{x+N^{2}\right\}$ can be expanded to a basis of $N / N^{2}$ over $\Lambda / N$. By Nakayama's Lemma, this basis can be lifted to a minimal generating set $\left\{x_{1}, \ldots, x_{t}\right\}$ for $N$, where $x_{1}=x$. The map $\Lambda \rightarrow N$ given by $\lambda \rightarrow \lambda x$ induces a homomorphism $g: \Lambda / N \rightarrow \bar{N}$. Now let $y \in N . \quad y=\sum_{i=1}^{t} \lambda_{i} x_{i}$. Suppose $\sum_{i=1}^{t} \lambda_{i} x_{i}=\sum_{i=1}^{t} \lambda_{i}^{\prime} x_{i}$. Then $\sum_{i=1}^{t}\left(\lambda_{i}-\lambda_{i}^{\prime}\right) x_{i}=0$. If for some $i, \lambda_{i}-\lambda_{i}^{\prime} \notin N$, then $\lambda_{i}-\lambda_{i}^{\prime}$ is a unit in $\Lambda$ and thus $x_{i} \in \Lambda\left(x_{1}, \ldots, x_{i-1}, \hat{x}_{i}, x_{i+1}, \ldots, x_{t}\right)$ (where denotes omission) contradicting the minimality of $\left\{x_{1}, \ldots, x_{t}\right\}$. So for all $i, \lambda_{i}-\lambda_{i}^{\prime}$ $\in N$. In particular, since $\lambda_{1}-\lambda_{1}^{\prime} \in N$, we have a map $h: N \rightarrow \Lambda / N$ given by $\sum_{i=1}^{t} \lambda_{i} x_{i}$ $\rightarrow \lambda_{1}+N$. 
$x N \subset \operatorname{Ker} h$. Thus we get a map $\bar{h}: \bar{N} \rightarrow \Lambda / N$. It is easily seen that $\bar{h} \circ g: \Lambda / N$ $\rightarrow \Lambda / N$ is the identity. Hence $\Lambda / N$ is a $\bar{\Lambda}$-direct summand of $\bar{N}$.

TheORem 4.2. Let $(R, \underline{m})$ be regular local of dimension $n$. Assume that $\Lambda$ is local, $\Lambda \supset R$ and $x \in \underline{m}-N^{2}$. Suppose $\mathrm{gl} \operatorname{dim} \Lambda<\infty$. Then $\mathrm{gl} \operatorname{dim} \Lambda=n, \Lambda$ is $R$-free, and $\mathrm{gl} \operatorname{dim} \bar{\Lambda}=n-1$.

Proof. The first two statements come from Corollary 2.18. By Corollary 1.3 $\operatorname{pd}_{\Lambda} \Lambda / N=\mathrm{gl} \operatorname{dim} \Lambda=n$. Thus $\operatorname{pd}_{\Lambda} N=n-1(n>0$ since $\underline{m} \neq(0)) . R$ is a domain, so $x$ is regular on $R$, and since $\Lambda$ is $R$-free, $x$ is regular on $\Lambda$. Hence $x$ is regular on $N$. By Corollary $2.2 \operatorname{pd}_{\bar{\Lambda}} \bar{N}=\operatorname{pd}_{\Lambda} N=n-1$. Since $\Lambda / N$ is a $\bar{\Lambda}$-direct summand of $\bar{N}$, $\operatorname{pd}_{\bar{\Lambda}} \Lambda / N \leqq \operatorname{pd}_{\bar{\Lambda}} \bar{N}=n-1$.

But $\operatorname{gl} \operatorname{dim} \bar{\Lambda}=\operatorname{pd}_{\bar{\Lambda}} \Lambda / N(\Lambda / N=\bar{\Lambda} / \bar{N})$.

So $\mathrm{gl} \operatorname{dim} \bar{\Lambda} \leqq n-1$, and thus by Corollary $2.18 \mathrm{gl} \operatorname{dim} \bar{\Lambda}=n-1$.

5. Maximal orders in dimension 2. Auslander and Goldman [4] have given a complete description of maximal orders over regular local rings $R$ of dimension 1 (i.e., discrete valuation rings). Three of their theorems are of particular interest to us in this section.

[4, TheOREM 2.3]. An $R$-order $\Lambda$ in a simple algebra $\Sigma$ is maximal if and only if $\Lambda$ is quasi-local and $\mathrm{gl} \operatorname{dim} \Lambda=1$.

[4, Proposition 2.5]. An order $\Lambda$ is maximal over $R$ (if and) only if $\hat{\Lambda}$ is maximal over $\hat{R}$.

(The "if" part of this theorem depends only on the fact that $\hat{R}$ is faithful on finitely generated $R$-modules, and is therefore true for any noetherian integrally closed local domain.)

[4, Proposition. 3.5]. Any two maximal orders over $R$ in the same simple algebra are isomorphic under an inner automorphism of the simple algebra.

In this section we examine the analogues of these theorems when $R$ is regular local of dimension 2. But we begin in a more general setting.

Let $R$ be an integrally closed noetherian domain with quotient field $K$. We denote by $P$ the set of all height one primes $p$ of $R . \Sigma$ is a central simple $K$-algebra with $[\Sigma: K]<\infty . \Lambda$ and $\Gamma$ are $R$-orders in $\Sigma$. If $M$ is an $R$-module, $M^{*}=\operatorname{Hom}_{R}(M, R)$.

" $M=M^{* *}$ " will mean that $M$ is $R$-reflexive, i.e., that the canonical map $M \rightarrow M^{* *}$ is an isomorphism.

LEMMA 5.1. Suppose $M$ is a finitely generated torsion-free $R$-module. Then $M^{* *}=\bigcap_{p \in P} M_{p}$. Consequently $M$ is $R$-reflexive if and only if $M=\bigcap_{p \in P} M_{p}$.

Proof. This is [7, $\S 4$, No. 2, Theorems 1 and 2].

Corollary 5.2. Suppose $A \subset B$ are finitely generated torsion-free $R$-modules and $A=A^{* *}$. If $A_{p}=B_{p}$ for all $p \in P$ then $A=B$. 
Proof. $B \subset B^{* *}=\bigcap_{p \in P} B_{p}=\bigcap_{p \in P} A_{p}=A^{* *}=A$. So $B \subset A$ and therefore $A=B$.

We recall the notion of the conductor:

Definition. The right conductor of $\Gamma$ in $\Lambda$, denoted by $C_{\Lambda}^{\mathrm{r}}(\Gamma)$, is $\{z \in \Lambda \mid \Gamma z \subset \Lambda\}$. $C_{\Lambda}^{\mathrm{r}}(\Gamma)$ is a right ideal in $\Lambda$ and a left $\Gamma$-module. Since $\Gamma \otimes_{R} K=\Sigma$, we have $\Gamma \subset \Lambda \otimes_{R} K . \Gamma$ is finitely generated as an $R$-module, so there is an $r \neq 0$ in $R$ such that $\operatorname{\Gamma r} \subset \Lambda$. In other words, $C_{\Lambda}^{\mathrm{r}}(\Gamma) \cap R \neq(0)$.

Proposition 5.3. If $\Lambda$ is $R$-reflexive, so is $C_{\Lambda}^{\mathrm{r}}(\Gamma)$.

Proof. By Lemma 5.1 it suffices to show that

$$
C_{\Lambda}^{\mathrm{r}}(\Gamma)=\bigcap_{p \in P}\left(C_{\Lambda}^{\mathrm{r}}(\Gamma) \otimes_{R} R_{p}\right)
$$

Clearly the left side is contained in the right side. Let $y \in \bigcap_{p \in P}\left(C_{\Lambda}^{\mathrm{r}}(\Gamma) \otimes_{R} R_{p}\right)$. Then for any $p \in P$ there is an $s \notin p$ such that $y s \in C_{\Lambda}^{\mathrm{r}}(\Gamma)$. Thus $\Gamma s y \subset \Lambda$ and so $(\Gamma y+\Lambda) \cdot s \subset \Lambda$. Since $\Lambda \subset \Gamma y+\Lambda$ we have $\Lambda_{p}=(\Gamma y+\Lambda)_{p}$. This is true for all $p \in P$, and $\Lambda=\Lambda^{* *}$ by hypothesis, so by Corollary $5.2 \Lambda=\Gamma y+\Lambda$. Thus $y \in C_{\Lambda}^{\mathrm{r}}(\Gamma)$. Hence $C_{\Lambda}^{\mathrm{r}}(\Gamma)=\bigcap_{p \in P}\left(C_{\Lambda}^{\mathrm{r}}(\Gamma) \otimes_{R} R_{p}\right)=C_{\Lambda}^{\mathrm{r}}(\Gamma)^{* *}$.

THEOREM 5.4. Let $R$ be a regular local ring of dimension two. Suppose the $R$-order $\Lambda$ in $\Sigma$ is quasi-local and $\mathrm{gl} \operatorname{dim} \Lambda<\infty$. Then if $\Gamma$ is any maximal $R$-order in $\Sigma$, $\Lambda=t^{-1} \Gamma t$ for some unit $t \in \Sigma$. Thus $\Lambda$ is a maximal order, and all maximal $R$-orders in $\Sigma$ are isomorphic. In particular, they are all quasi-local and have global dimension two.

Proof. Since $\Lambda$ is quasi-local and $\mathrm{gl} \operatorname{dim} \Lambda<\infty$, by Corollary $2.17 \Lambda$ is $R$-free and $\mathrm{gl} \operatorname{dim} \Lambda=2$. Since $\Lambda$ is $R$-free, $\Lambda=\Lambda^{* *}$. Thus if $\Gamma$ is any $R$-order in $\Sigma$, $C_{\Lambda}^{\mathrm{r}}(\Gamma)$ is $R$-reflexive by the preceding proposition. But $\mathrm{gl} \operatorname{dim} R=2$, so by [4, Corollary to Proposition 4.7] $C_{\Lambda}^{\mathrm{r}}(\Gamma)$ is $R$-free. By Proposition $3.5 C_{\Lambda}^{\mathrm{r}}(\Gamma)$ is $\Lambda$ projective. Thus $C_{\Lambda}^{\mathrm{r}}(\Gamma)$ is a projective right ideal in $\Lambda$, and hence principal by [4, Proposition 3.3], since $\Lambda$ is quasi-local and $R$-free. So $C_{\Lambda}^{\mathrm{r}}(\Gamma)=t \Lambda$ for some $t \in \Lambda$. We conclude the argument by using the proof of [4, Lemma 3.4]. Since $C_{\Lambda}^{\mathrm{r}}(\Gamma) \cap R \neq(0), t$ is a unit in $\Sigma$. We have $\Gamma t \subset C_{\Lambda}^{\mathrm{r}}(\Gamma)=t \Lambda$ since $C_{\Lambda}^{\mathrm{r}}(\Gamma)$ is a left $\Gamma$-module. Therefore $\Gamma \subset t \Lambda t^{-1}$. Now if $\Gamma$ is a maximal order, $\Gamma=t \Lambda t^{-1}$.

Since a regular local ring is a unique factorization domain, the intersection of any two principal ideals is again principal. If $R$ is regular local of dimension two and $\Lambda$ is an $R$-order it seems natural to inquire under what conditions $\Lambda$ will inherit this property.

Proposition 5.5. Let $R$ be regular local of dimension two and $\Lambda$ an $R$-free $R$ order in $\Sigma$ with $\mathrm{gl} \operatorname{dim} \Lambda=2$. Then:

(1) The intersection of two $\Lambda$-projective left ideals is $\Lambda$-projective.

(2) If $\Lambda$ is quasi-local the intersection of two (principal) $\Lambda$-projective left ideals is principal. 
Hence if $\Sigma$ is a division ring and $\Lambda$ is a quasi-local $R$-order with $\mathrm{gl} \operatorname{dim} \Lambda=2$, the intersection of any two principal left ideals of $\Lambda$ is principal.

Proof. (1) Suppose $I$ and $J$ are projective left ideals in $\Lambda$. Then $I$ and $J$ are both $R$-reflexive since $\Lambda$ is. Using Lemma 5.1 it is easy to see that $I \cap J$ is $R$-reflexive, since for any prime $p,(I \cap J)_{p}=I_{p} \cap J_{p}$. But since $\mathrm{gl} \operatorname{dim} R=2, I \cap J$ must be

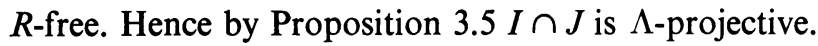

(2) This follows from the fact that if $\Lambda$ is quasi-local and $R$-free, then every projective ideal is principal [4, Proposition 3.3].

Finally, if $\Sigma$ is a division ring, then a principal ideal in $\Lambda$ is $\Lambda$-free, and a fortiori $\Lambda$-projective.

Theorem 5.4 generalizes half of [4, Theorem 2.3] to regular local rings of dimension two. We shall now construct examples to show that neither the other half of [4, Theorem 2.3] nor [4, Proposition 2.5] generalizes.

Let $K$ be a field whose characteristic is different from 2. We define a $K$-algebra $\Sigma$ as follows: $\Sigma=K[1, \alpha, \beta, \alpha \beta]$ (i.e., $\{1, \alpha, \beta, \alpha \beta\}$ is a vector space basis of $\Sigma$ over $K$ ) where $\beta \alpha=-\alpha \beta, \alpha^{2}=w, \beta^{2}=v, w, v \in K-\{0\}$. Furthermore, for any $x \in K, x \alpha=\alpha x, x \beta=\beta x$.

$\Sigma$ is called a quaternion algebra over $K$. Since $w$ and $v$ are different from zero, $\Sigma$ is a central simple $K$-algebra. For if $v^{1 / 2} \notin K, \Sigma$ is the crossed-product algebra formed from the cyclic extension $K\left(v^{1 / 2}\right) / K$, whose Galois group is generated by $\sigma$, and the 2-cocycle $f$ determined by $f(\sigma, \sigma)=w$. On the other hand, if $v^{1 / 2} \in K$, then $\Sigma \approx M_{2}(K)$, the ring of $2 \times 2$ matrices over $K$, which is central simple over $K$. The $K$-algebra isomorphism is given by

$$
\alpha \rightarrow\left[\begin{array}{ll}
0 & w \\
1 & 0
\end{array}\right], \quad \beta \rightarrow\left[\begin{array}{cc}
v^{1 / 2} & 0 \\
0 & -v^{1 / 2}
\end{array}\right]
$$

In any case, $\Sigma$ is central simple over $K$, so by Wedderburn's Theorem $\Sigma \approx$ $\operatorname{End}_{\Delta}(V)$ where $\Delta$ is a division ring whose center is $K$ and $V$ is a finite-dimensional vector space over $\Delta .4=[\Sigma: K]=[V: \Delta]^{2} \cdot[\Delta: K]$.

Hence $[\Delta: K]=1$ or 4 . Thus either $\Sigma=\Delta$ or $\Sigma=\operatorname{End}_{K}(V)$, i.e., $\Sigma$ is either a division ring or $M_{2}(K)$.

Suppose $w$ and $v$ lie in $R$, a domain with quotient field $K$. Let $\Lambda$ be the $R$ module generated by $1, \alpha, \beta, \alpha \beta$. Then $\Lambda$ is free on these generators and is closed under multiplication since $\alpha^{2}$ and $\beta^{2}$ are in $R$. Thus $\Lambda$ is an $R$-free $R$-order in $\Sigma$. $\Lambda$ may or may not be maximal, depending on $w, v$, and $R$ as we shall see.

EXAMPLE 1. Let $F$ be any field with characteristic unequal to 2 . Let $R=$ $F[X, Y]_{(X, Y)}$, where $X$ and $Y$ are indeterminates over $F$. Then $R$ is regular local of dimension two and has quotient field $K=F(X, Y)$. The maximal ideal $\underline{m}=(X, Y)$. $\hat{R}=F[[X, Y]]$. Let $R=F((X, Y))$, the quotient field of $R$. (In this case $R \neq$ $K \otimes_{R} \hat{R}$.) 
Define a quaternion algebra $\Sigma$ over $K$ by taking $w=X$ and $v=1+Y$. In other words, $\Sigma=K[1, \alpha, \beta, \alpha \beta]$ where $\beta \alpha=-\alpha \beta, \alpha^{2}=X$, and $\beta^{2}=1+Y$. Let $\Lambda$ be the $R$-free order $R[1, \alpha, \beta, \alpha \beta]$.

To establish the maximality of $\Lambda$ it suffices, by [4, Theorem 1.5], to show that $\Lambda_{p}$ is a maximal $R_{p}$-order in $\Sigma$ for all $p \in P$. By [4, Theorem 2.3] $\Lambda_{p}$ is maximal if and only if it is quasi-local and gl $\operatorname{dim} \Lambda_{p}=1$.

If $p \neq(X)$, then $\Lambda_{p} / p \Lambda_{p}$ is a quaternion algebra over $R_{p} / p R_{p}$ and is therefore a simple ring. Thus $p \Lambda_{p}=\operatorname{Rad} \Lambda_{p}$ and $\Lambda_{p}$ is quasi-local. Moreover, $p=(z) R$ for some $z \in R$ since $R$ is a UFD. Hence $\operatorname{Rad} \Lambda_{p}=(z) \Lambda_{p}$ is $\Lambda_{p}$-free and therefore gl $\operatorname{dim} \Lambda_{p}=1$. Thus $\Lambda_{p}$ is maximal.

Now let $p=(X)$. Since the image of $\alpha$ in $\Lambda_{(X)} / X \Lambda_{(X)}$ is nilpotent and generates a two-sided ideal, it belongs to the radical, and therefore $\alpha \in \operatorname{Rad} \Lambda_{(X)} . \Lambda_{(X)} / \alpha \Lambda_{(X)}$ $\approx F(Y)[T] /\left(T^{2}-(1+Y)\right)$ where $T$ is an indeterminate.

The latter is a field, for $1+Y$ is not a square in $F[Y]$ (its degree is odd) and hence it is not a square in $F(Y)$ since $F[Y]$ is integrally closed in $F(Y)$. Thus $\operatorname{Rad} \Lambda_{(X)}$ $=\alpha \Lambda_{(X)}$, which is $\Lambda_{(X)}$-free, and $\Lambda_{(X)}$ is local. Hence $\mathrm{gl} \operatorname{dim} \Lambda_{(X)}=1$ and $\Lambda_{(X)}$ is maximal.

So for all $p \in P, \Lambda_{p}$ is maximal and therefore $\Lambda$ is maximal. Furthermore, $\Sigma$ is a division ring by [4, Theorem 3.11] since the maximal $R_{(X)}$-order $\Lambda_{(X)}$ is local.

Reasoning as before we see that $\alpha \in \operatorname{Rad} \Lambda$. Hence $(\alpha, Y) \Lambda \subset \operatorname{Rad} \Lambda$.

$$
\Lambda /(\alpha, Y) \Lambda \simeq F[T] /\left(T^{2}-1\right) \approx F[T] /(T-1) \oplus F[T] /(T+1) .
$$

So $\Lambda /(\alpha, Y) \Lambda \approx F \oplus F$ and is thus semisimple. Hence $\operatorname{Rad} \Lambda=(\alpha, Y) \Lambda$. Furthermore, from the above decomposition we see that $\Lambda(\alpha, \beta-1)$ and $\Lambda(\alpha, \beta+1)$ are the maximal two-sided ideals of $\Lambda$, and $\Lambda$ is not quasi-local.

In order to show that $\mathrm{gl} \operatorname{dim} \Lambda=2$ we shall establish a general result.

Proposition 5.6. Let $(R, \underline{m})$ be a noetherian local ring, $\Lambda$ an $R$-algebra, finitely generated as an $R$-module. If $z \in \underline{m}$ is regular on $\Lambda$ and $\mathrm{gl} \operatorname{dim} \Lambda / z \Lambda=k<\infty$, then $\operatorname{gl} \operatorname{dim} \Lambda=k+1$.

Proof. Let $\bar{\Lambda}=\Lambda / z \Lambda, N=\operatorname{Rad} \Lambda$, and $\bar{N}=N / z N . z$ is regular on $N$ since it is regular on $\Lambda$, and so. by Corollary $2.2 \operatorname{pd}_{\Lambda} N=\operatorname{pd}_{\bar{\Lambda}} \bar{N}$. Thus $\operatorname{pd}_{\Lambda} N \leqq K$, which means that $\mathrm{gl} \operatorname{dim} \Lambda=\operatorname{pd}_{\Lambda} \Lambda / N \leqq k+1$. Therefore

$$
\operatorname{gl} \operatorname{dim} \Lambda=\operatorname{inj} \operatorname{dim}_{\Lambda} \Lambda=1+\operatorname{inj} \operatorname{dim}_{\bar{\Lambda}} \bar{\Lambda}=1+\operatorname{gl} \operatorname{dim} \bar{\Lambda}=k+1
$$

Returning to our example, it is thus sufficient to show that $\mathrm{gl} \operatorname{dim} \Lambda / Y \Lambda=1$. Since $\operatorname{Rad} \Lambda=\Lambda(\alpha, Y), \operatorname{Rad}(\Lambda / Y \Lambda)=\Lambda / Y \Lambda(\bar{\alpha}) . \bar{\alpha}$ is regular on $\Lambda / Y \Lambda$ since $\bar{\alpha}^{2}=\bar{X}$ which is regular on $\Lambda / Y \Lambda$. Thus $\operatorname{Rad}(\Lambda / Y \Lambda)$ is free over $\Lambda / Y \Lambda$ and so $\mathrm{gl} \operatorname{dim} \Lambda / Y \Lambda=1$. Hence $\mathrm{gl} \operatorname{dim} \Lambda=2$.

Next we show that $\hat{\Sigma}$ (i.e., $\Sigma \otimes_{K} \hat{K}$ ) is the full matrix ring $M_{2}(\hat{K})$. Since characteristic $F \neq 2,1+Y$ has a square root in $F[[Y]] \subset F((X, Y))=R$. Thus, as we 
remarked earlier,

$$
\alpha \rightarrow\left[\begin{array}{cc}
0 & X \\
1 & 0
\end{array}\right], \quad \beta \rightarrow\left[\begin{array}{cc}
(1+Y)^{1 / 2} & 0 \\
0 & -(1+Y)^{1 / 2}
\end{array}\right]
$$

determines a $\hat{R}$-algebra isomorphism between $\hat{\Sigma}$ and $M_{2}(\hat{K})$.

Since $\hat{\Lambda} / \operatorname{Rad} \hat{\Lambda} \simeq \Lambda / \operatorname{Rad} \Lambda, \hat{\Lambda}$ is not quasi-local. But by [4, Theorem 4.3] any maximal $R$-order in $M_{2}(\hat{K})$ is of the form $\operatorname{End}_{\hat{R}}(E)$ where $E$ is $\hat{R}$-free of rank two. In particular, any maximal $\hat{R}$-order in $M_{2}(\hat{R})$ is quasi-local. Hence $\hat{\Lambda}$ is a nonmaximal $\hat{R}$-order in $\hat{\Sigma}$. $\mathrm{gl} \operatorname{dim} \hat{\Lambda}=\mathrm{gl} \operatorname{dim} \Lambda=2$.

In summary, $\Lambda$ is a maximal $R$-order in a division ring $\Sigma$. While $\mathrm{gl} \operatorname{dim} \Lambda=2$, $\Lambda$ is not quasi-local. Its completion $\hat{\Lambda}$ is a nonmaximal $\hat{R}$-order in a full matrix ring $\hat{\Sigma}$ over $\hat{K}$.

We shall now prove a theorem which will be used to establish our second example.

THEOREM 5.7. Let $(R, \underline{m})$ be regular local of dimension two. $\Lambda$ is an $R$-order in $\Sigma$. Suppose $\mathrm{gl} \operatorname{dim} \Lambda<\infty, \Lambda$ is quasi-local, center of $\Lambda / \operatorname{Rad} \Lambda=R / \underline{m}, R / \underline{m}$ is perfect, and $\underline{m} \leftarrow N^{2}$, where $N=\operatorname{Rad} \Lambda$. Then $\Lambda$ is separable over $R$.

REMARK. The proof of this theorem depends upon the fact that if $z \in \underline{m}-N^{2}$, then $\mathrm{gl} \operatorname{dim} \Lambda / z \Lambda=\mathrm{gl} \operatorname{dim} \Lambda-1$. This was proved in Theorem 4.2, assuming $\Lambda$ local. The result will be extended to quasi-local rings in the next section, but using it here involves no circularity.

Proof. Since $\underline{m} \notin N^{2}, \underline{m} \supsetneqq \underline{m} \cap N^{2} \supset \underline{m}^{2}$.

Claim: There are infinitely many distinct prime ideals $(z)$ in $R$ with $z \in \underline{m}-N^{2}$. For let $x \in \underline{m}-N^{2}$. Then $x \in \underline{m}-\underline{m}^{2}$ and since $\operatorname{gl} \operatorname{dim} R=2$ there is a $y \in \underline{m}-\underline{m}^{2}$ such that $\underline{m}=(x, y)$, i.e., $x$ and $y$ form a minimal system of generators of $\underline{m}$. For $n \geqq 2, x+y^{n} \in \underline{m}-N^{2} \subset \underline{m}-\underline{m}^{2}$, and so $\left(x+y^{n}\right)$ is prime. It is easily seen that these ideals are all distinct.

Denote by $h(\Lambda / R)$ the homological different of $\Lambda$ over $R$ (see $[5$, p. 378]). $h(\Lambda / R)$ $\otimes_{R} K=h(\Sigma / K) \neq 0$ since $\Sigma$ is separable over $K$. Thus $h(\Lambda / R)$ is a nonzero ideal of $R$, and since $R$ is noetherian, only finitely many prime ideals $(z)$ can contain $h(\Lambda / R)$. Therefore there exists a prime ideal $(z)$ such that $z \in \underline{m}-N^{2}$ and $(z) \ngtr h(\Lambda / R)$. Hence $\Lambda_{(z)}$ is $R_{(z)}$-separable by [5, Corollary 4.3]. The center of $\Lambda$ is $R$, so that $\Lambda_{(z)}$ is central $R_{(z)}$-separable. Thus $\Lambda_{(z)} / z \Lambda_{(z)}$ is central separable over $R_{(z)} / z R_{(z)}$ by [5, Proposition 1.4].

Since $\mathrm{gl} \operatorname{dim} \Lambda<\infty$ and $\Lambda$ is quasi-local, $\Lambda$ is $R$-free and $\mathrm{gl} \operatorname{dim} \Lambda=2$ by Corollary 2.17. Since $z \in \underline{m}-N^{2}, \operatorname{gl} \operatorname{dim} \Lambda / z \Lambda=1$ (see the above Remark). $\Lambda / z \Lambda$ is quasi-local. Now $R / z$ is a discrete valuation ring because $\mathrm{gl} \operatorname{dim} R / z=\mathrm{gl} \operatorname{dim} R-1$ $=1$. Thus by [4, Theorem 2.3] $\Lambda / z \Lambda$ is a maximal $R / z$-order in $\Lambda_{(z)} / z \Lambda_{(z)}$. Furthermore $\Lambda / z \Lambda / N / z \Lambda \simeq \Lambda / N$ and $R /(z) / \underline{m} /(z) \simeq R / \underline{m}$. Hence center of $\Lambda / z \Lambda / N / z \Lambda$ $=R /(z) / \underline{m} /(z)$, which is a perfect field. Thus the hypotheses of [5, Proposition 8.4] 
are satisfied and so $\Lambda / z \Lambda$ is $R / z$-separable. Therefore $\Lambda / \underline{m} \Lambda$ is $R / \underline{m}$-separable [5, Proposition 1.4]. By [5, Proposition 4.7] $\Lambda$ is $R$-separable.

EXAMPLE 2. Let $k$ be a perfect field of characteristic $\neq 2$. Let $R=k[[X, Y]], X$ and $Y$ transcendental over $k . K=k((X, Y)) . \underline{m}=(X, Y) .(R, \underline{m})$ is a complete regular local ring of dimension two.

Let $\Sigma$ be the quaternion algebra $K[1, \alpha, \beta, \alpha \beta]$ where $\alpha^{2}=X$ and $\beta^{2}=Y(Y-X)$ . $(Y+X)$. Let $\Lambda=R[1, \alpha, \beta, \alpha \beta] . \Lambda$ is an $R$-free order in $\Sigma$. The maximality of $\Lambda$ is proved just as in Example 1. Again, $\Lambda_{(x)} / \alpha \Lambda_{(x)}$ is a field. So the maximal $R_{(x)^{-}}$ order $\Lambda_{(x)}$ is local and thus $\Sigma$ is a division $\operatorname{ring} \operatorname{Rad} \Lambda=N=\Lambda(\alpha, \beta, Y)$ and so $\Lambda / N \simeq R / \underline{m}=k$. Therefore $\Lambda$ is local. Since $N \supsetneqq \underline{m} \Lambda, \Lambda / \underline{m} \Lambda$ is not a simple ring and thus not $R / \underline{m}$-separable. Hence $\Lambda$ is not separable over $R$.

$\Lambda / \alpha \Lambda \approx k[[Y]][T] /\left(T^{2}-Y^{3}\right)=S . S$ is a local domain with maximal ideal $J$ $=(\bar{Y}, \bar{T})$. Now suppose $Y \in N^{2}$. Then $\bar{Y} \in J^{2}$ and so by Nakayama's Lemma $J=(\bar{T})$. Thus $\bar{Y} \in(\bar{T})$, i.e., $Y-f \cdot T=g \cdot\left(T^{2}-Y^{3}\right)$ for some $f, g \in k[[Y]][T]$. This means that $T$ divides $Y\left(1+g Y^{2}\right)$, and since $T$ does not divide $Y$ it must divide $1+g Y^{2}$.

Let $\varphi: k[[Y]][T] \rightarrow k[[Y]]$ by $\varphi(T)=Y$ and $\varphi=$ identity on $k[[Y]]$. Then in $k[[Y]] Y$ divides the unit $1+\varphi(g) Y^{2}$. This is impossible, so $Y \notin N^{2}$.

Thus $\underline{m} \notin N^{2} . \Lambda$ is local and center of $\Lambda / N=R / \underline{m}$, which is perfect. Since $\Lambda$ is not separable over $R$ we conclude from Theorem 5.7 that $\mathrm{gl} \operatorname{dim} \Lambda=\infty$.

Summarizing this example: The division ring $\Sigma$ contains a maximal $R$-order $\Lambda$ which is local but has infinite global dimension.

REMARK. inj $\operatorname{dim}_{\Lambda} \Lambda=2$.

For let $\Gamma=R[1, \sigma, \tau, \sigma \tau]$ where $\sigma^{2}=X, \tau^{2}=Y$, and $\tau \sigma=-\sigma \tau$. Then $\operatorname{Rad} \Gamma$ $=\Gamma(\sigma, \tau)$ and

$$
0 \rightarrow \Gamma\langle\tau, \sigma\rangle \rightarrow \Gamma \oplus \Gamma \rightarrow \Gamma(\sigma, \tau) \rightarrow 0
$$

is a free $\Gamma$-resolution of $\operatorname{Rad} \Gamma$, where the map $\Gamma \oplus \Gamma \rightarrow \Gamma(\sigma, \tau)$ is given by $\left\langle\gamma_{1}, \gamma_{2}\right\rangle \rightarrow \gamma_{1} \sigma+\gamma_{2} \tau$. So $\operatorname{pd}_{\Gamma} \operatorname{Rad} \Gamma=1$ and $g l \operatorname{dim} \Gamma=2$. Therefore inj $\operatorname{dim}_{\Gamma} \Gamma=2$. $\Gamma$ is $R$-free, so $X, Y$ is a regular $\Gamma$-sequence. It follows from Theorem 2.10 that inj $\operatorname{dim}_{\Gamma / m \Gamma} \Gamma / \underline{m} \Gamma=0$. But $\Gamma / \underline{m} \Gamma \approx \Lambda / \underline{m} \Lambda$. Thus $\operatorname{inj} \operatorname{dim}_{\Lambda / m \Lambda} \Lambda / \underline{m} \Lambda=0$ and using Theorem 2.10 again, since $X, Y$ is a regular $\Lambda$-sequence, $\operatorname{inj} \operatorname{dim}_{\Lambda} \Lambda=2$.

6. Endomorphism rings and Morita duality. $R$ is an integrally closed noetherian domain.

LEMMA 6.1. Let $\Gamma$ be any R-algebra and let $E$ and $M$ be $\Gamma$-modules (both left or both right) which are finitely generated as $R$-modules. Suppose $M$ is $R$-reflexive. Then $\operatorname{Hom}_{\Gamma}(E, M)$ is $R$-reflexive.

Proof. Let $F_{1} \rightarrow F_{0} \rightarrow E \rightarrow 0$ be exact with the $F_{i}$ finitely generated free $\Gamma$ modules. Applying $\operatorname{Hom}_{\Gamma}(-, M)$ we get an exact sequence of $R$-modules

$$
0 \rightarrow \operatorname{Hom}_{\Gamma}(E, M) \rightarrow \operatorname{Hom}_{\Gamma}\left(F_{0}, M\right) \rightarrow \operatorname{Hom}_{\Gamma}\left(F_{1}, M\right) .
$$


Since $F_{i}$ is $\Gamma$-free, $\operatorname{Hom}_{\Gamma}\left(F_{i}, M\right)$ is isomorphic to a direct sum of copies of the reflexive $R$-module $M$ and is therefore $R$-reflexive. Now in [2, Lemma 2] several equivalent conditions are given for reflexivity of a finitely generated module over an integrally closed noetherian domain. Another condition can be added and proved equivalent to the others by a proof nearly identical to the proof in [2, Lemma 2] that "(d) $\Rightarrow$ (e)". The condition on a module $A$ is:

There exists an exact sequence $0 \rightarrow A \rightarrow G_{0} \rightarrow G_{1}$ with the $G_{i} R$-reflexive.

Using this we see that $\operatorname{Hom}_{\Gamma}(E, M)$ is $R$-reflexive.

For the rest of this section $\Sigma$ will denote a central simple $K$-algebra, where $K$ is the quotient field of $R$.

THEOREM 6.2. Let $\Lambda$ and $\Gamma$ be R-orders in $\Sigma$, with $\Gamma$ maximal. Then $\Gamma=$ $\operatorname{End}_{\Lambda}\left(C_{\Lambda}^{\mathrm{r}}(\Gamma)\right)$. If $J$ is any $R$-reflexive right ideal in $\Gamma$ with $J \cap R \neq(0)$ then $\operatorname{End}_{\Gamma}(J)$ is a maximal $R$-order in $\Sigma$.

Proof. $C_{\Lambda}^{\mathrm{r}}(\Gamma)$ is a left $\Gamma$-module, so we have a map $\Gamma \rightarrow \operatorname{End}_{\Lambda}\left(C_{\Lambda}^{\mathrm{r}}(\Gamma)\right)$ given by $\gamma \rightarrow(z \rightarrow \gamma z)$. Since $C_{\Lambda}^{\mathrm{r}}(\Gamma) \cap R \neq(0)$ and $\Gamma$ is $R$-torsion-free, this map is injective. In fact, by means of the identification of $\operatorname{End}_{\Sigma}(\Sigma)$ with $\Sigma$ (regarding $\Sigma$ as a right $\Sigma$-module), $\operatorname{End}_{\Lambda}\left(C_{\Lambda}^{\mathrm{r}}(\Gamma)\right)$ is an $R$-order in $\Sigma$ containing $\Gamma$. But $\Gamma$ is maximal, so $\Gamma=\operatorname{End}_{\Lambda}\left(C_{\Lambda}^{\mathbf{r}}(\Gamma)\right)$.

For the second statement, the fact that $J \cap R \neq(0)$ implies that $K \otimes_{R} J=\Sigma$ and so $\operatorname{End}_{\Gamma}(J)$ is an $R$-order in $\Sigma$. By Lemma 6.1 $\operatorname{End}_{\Gamma}(J)$ is $R$-reflexive since $J$ is. Now let $p \in P$, i.e., $p$ is a height one prime ideal of $R$. $\operatorname{End}_{p}(J) \otimes_{R} R_{p} \simeq \operatorname{End}_{\Gamma_{p}}\left(J_{p}\right)$. $\Gamma_{p}$ is maximal over the discrete valuation ring $R_{p}$ since $\Gamma$ is maximal over $R$. Hence $\Gamma_{p}$ is a principal ideal ring by [4, Corollary to Proposition 3.3] Therefore $J_{p}=t \Gamma_{p}$ for some $t \in \Gamma_{p}$, and since $J_{p} \cap R_{p} \neq(0), t$ is a unit in $\Sigma$.

$\operatorname{End}_{\Gamma_{p}}\left(J_{p}\right)=\operatorname{End}_{\Gamma_{p}}\left(t \Gamma_{p}\right) \supset t \Gamma_{p} t^{-1}$. But $t \Gamma_{p} t^{-1}$ is maximal, so $\operatorname{End}_{\Gamma_{p}}\left(J_{p}\right)=t \Gamma_{p} t^{-1}$. Thus $\operatorname{End}_{\Gamma_{p}}\left(J_{p}\right)$ is a maximal $R_{p}$-order. So $\operatorname{End}_{\Gamma}(J)$ is an $R$-reflexive order whose localization at each $p \in P$ is maximal. Thus $\operatorname{End}_{\Gamma}(J)$ is maximal.

COROLlaRY 6.3. Let $\Lambda$ be a maximal $R$-order in $\Sigma$. Then $\Lambda$ is the only maximal $R$-order in $\Sigma$ if and only if every $R$-reflexive ideal $J$ in $\Lambda$ with $J \cap R \neq(0)$ is two-sided.

Proof. By Theorem 6.2 the maximal $R$-orders in $\Sigma$ are precisely the rings $\operatorname{End}_{\Lambda}(J)$ where $J$ ranges over all $R$-reflexive right ideals $I$ such that $I \cap R \neq(0)$. $\operatorname{End}_{\Lambda}(J)=\{x \in \Sigma \mid x J \subset J\} . J$ is two-sided if and only if $\Lambda \subset \operatorname{End}_{\Lambda}(J)$. Since $\Lambda$ is maximal, this is true if and only if $\Lambda=\operatorname{End}_{\Lambda}(J)$. Thus $\Lambda$ is the unique maximal $R$-order in $\Sigma$ if and only if every $R$-reflexive right ideal $J$ in $\Lambda$ with $J \cap R \neq(0)$ is two-sided.

Now suppose $\Lambda$ is unique and $J$ is an $R$-reflexive left ideal in $\Lambda$ such that $J \cap R$ $\neq(0)$. Let $\Sigma^{0}$ be the opposite algebra of $\Sigma$. Then $\Lambda^{0}$ is the unique maximal $R$-order in $\Sigma^{0} . J^{0}$ is an $R$-reflexive right ideal in $\Lambda^{0}$ and $J^{0} \cap R \neq(0)$. Hence $J^{0}$ is two-sided in $\Lambda^{0}$. Thus $J$ is two-sided in $\Lambda$. 
We now wish to strengthen [4, Theorem 3.11], which gives three equivalent conditions for the uniqueness of the maximal $R$-order $\Lambda$ when $R$ is a discrete valuation ring.

Proposition 6.4. Let $R$ be a discrete valuation ring and $\Lambda$ a maximal $R$-order in $\Sigma$. The following are equivalent:

(a) $\Lambda$ is local.

(b) Every ideal in $\Lambda$ is two-sided and a power of $N=\operatorname{Rad} \Lambda$.

(c) $\Sigma$ is a division ring and $\Lambda$ is the only maximal $R$-order in $\Sigma$.

(d) $\Lambda$ is the only maximal $R$-order in $\Sigma$.

(e) Every right ideal $J$ in $\Lambda$ such that $J \cap R \neq(0)$ is two-sided.

Proof. The equivalence of (a), (b), and (c) is [4, Theorem 3.11].

(c) $\Rightarrow$ (d): Trivial.

(d) $\Leftrightarrow$ (e): This follows from Corollary 6.3 and the fact that every ideal in $\Lambda$ is $R$-free, hence $R$-reflexive.

(e) $\Rightarrow$ (a): Let $J$ be a right ideal in $\Lambda$ containing $N$. Then $J \cap R \supset N \cap R=\underline{m} \neq(0)$. Thus by our hypothesis $J$ is two-sided. But since $\Lambda$ is a maximal order over a discrete valuation ring, $\Lambda$ is quasi-local, i.e., $N$ is a maximal two-sided ideal. Thus $J=\Lambda$ or $J=N$. Hence $N$ is a maximal right ideal and so $\Lambda$ is local.

Auslander and Goldman [4] have proved the following structure theorem:

[4, THEOREM 3.6]. If $\Lambda$ is a maximal order over a discrete valuation ring $\mathcal{O}$ and $E$ is an indecomposable $\Lambda$-module which is finitely generated and torsion-free over $\mathcal{O}$, then $\Omega=\operatorname{End}_{\Lambda}(E)$ is a maximal order in a division algebra and $\Lambda=\operatorname{End}_{\Omega}(E)$. Conversely if $\Omega$ is a maximal order and $E$ is an $\Omega$-module which is finitely generated and torsion-free over $\mathcal{O}$, then $\operatorname{End}_{\Omega}(E)$ is a maximal order.

We now give a generalization of this to maximal orders over an arbitrary integrally closed noetherian domain $R$.

THEOREM 6.5. Suppose $\Lambda$ is a maximal $R$-order in $\Sigma$.

(a) Let $E$ be any finitely generated $R$-reflexive left $\Lambda$-module. Then $\Gamma=\operatorname{End}_{\Lambda}(E)$ is a maximal $R$-order in $\operatorname{End}_{\Sigma}(V)$, where $V=E \otimes_{R} K$. Furthermore, $\Lambda=\operatorname{End}_{\Gamma}(E)$.

(b) $E$ can be chosen so that $\operatorname{End}_{\Sigma}(V)$ is a division algebra $\Delta$. Thus $\Lambda=\operatorname{End}_{\Gamma}(E)$ for some maximal $R$-order $\Gamma$ in a division algebra $\Delta$.

Proof. (a) $\Gamma$ is $R$-reflexive by Lemma 6.1. For any $p \in P E_{p}$ is $R_{p}$-free and $\Lambda_{p}$ is maximal over $R_{p}$. The maximality of $\Gamma_{p}$ over $R_{p}$ now follows from [4, Theorem 3.6], stated above. Hence $\Gamma$ is a maximal $R$-order.

Since $E$ is a left $\Lambda$-module it is also a left $\operatorname{End}_{\Lambda}(E)$-module, i.e., a left $\Gamma$-module. The operations of $\Lambda$ and $\Gamma$ commute on $E$ and thus we get a ring homomorphism $\varphi: \Lambda \rightarrow \operatorname{End}_{\Gamma}(E)$ given by $\varphi(\lambda)(e)=\lambda e$.

$\varphi \otimes 1_{K}: \Lambda \otimes_{R} K \rightarrow \operatorname{End}_{\Gamma}(E) \otimes_{R} K$ is the isomorphism in the Wedderburn Theorem. By means of this isomorphism we may regard $\operatorname{End}_{\Gamma}(E)$ as an $R$-order 
in $\Sigma=\Lambda \otimes_{R} K$. Ker $\varphi \subset \operatorname{Ker}\left(\varphi \otimes 1_{K}\right)=0$. Thus $\operatorname{Ker} \varphi=0$ and $\Lambda \subset \operatorname{End}_{\Gamma}(E)$. But $\Lambda$ is maximal, so $\Lambda=\operatorname{End}_{\Gamma}(E)$.

(b) Let $V$ be a simple left $\Sigma$-module. By the Wedderburn $\operatorname{Theorem} \operatorname{End}_{\Sigma}(V)$ is a division ring $\Delta$ and $\Sigma=\operatorname{End}_{\Delta}(V)$. Let $v \in V, v \neq 0$, and let $M=\Lambda v$. Then $M \otimes_{R} K$ $=V$. Now let $E=M^{* *}$. $E$ is an $R$-reflexive left $\Lambda$-module since by Lemma 5.1 $E=\bigcap_{p \in P} M_{p}$ and $\Lambda \subset \Lambda^{* *}=\bigcap_{p \in P} \Lambda_{p}$ (in fact $\Lambda=\Lambda^{* *}$ since $\Lambda$ is maximal). Clearly $E \otimes_{R} K=M \otimes_{R} K=V$. By part (a) $\Gamma=\operatorname{End}_{\Lambda}(E)$ is a maximal $R$-order in $\Delta=$ $\operatorname{End}_{\Sigma}(V)$, and $\Lambda=\operatorname{End}_{\Gamma}(E)$.

For a closer investigation of the type of duality exhibited in Theorem 6.5 we need the Morita duality theory, a treatment of which can be found in [4, Appendix]. Here we shall give only a brief summary, without proofs, of those results we need.

Let $\Gamma$ be any ring and $E$ a left $\Gamma$-module. Let $\Omega=\operatorname{End}_{\Gamma}(E)$, As was mentioned before, $E$ is a left $\Omega$-module and there is a ring homomorphism $\varphi: \Gamma \rightarrow \operatorname{End}_{\Omega}(E)$. The trace ideal of $E$ in $\Gamma$, denoted by $\tau_{\Gamma}(E)$, is the two-sided ideal in $\Gamma$ generated by $\left\{f(e) \mid f \in \operatorname{Hom}_{\Gamma}(E, \Gamma), e \in E\right\}$.

Theorem. Suppose $\tau_{\Gamma}(E)=\Gamma$. Then

(a) $E$ is a finitely generated projective $\Omega$-module;

(b) $\Gamma \stackrel{\varphi}{\rightarrow} \operatorname{End}_{\Omega}(E)$ is an isomorphism.

Proposition. If $E$ is a finitely generated projective $\Gamma$-module then $\tau_{\Gamma}(E) \cdot E=E$ and $\tau_{\Omega}(E)=\Omega$.

DEFINITION. If $E$ is finitely generated $\Gamma$-projective and $\tau_{\Gamma}(E)=\Gamma$, then $\Gamma$ and $\Omega$ are Morita equivalent.

Note that by the preceding theorem and proposition this relation is symmetric. However, it is not reflexive, but "antireflexive". For $\Gamma$ is Morita-equivalent to $\operatorname{End}_{\Gamma}(\Gamma)$, considering $\Gamma$ as a left $\Gamma$-module. But since we stick to the usual convention for composing maps, whereby $f \circ g$ means "first apply $g$ and then $f$ ", we find that $\operatorname{End}_{\Gamma}(\Gamma)=\Gamma^{0}$.

Let mod- $\Gamma$ denote the category of right $\Gamma$-modules and $\Omega$-mod the category of left $\Omega$-modules. There are covariant additive functors

$$
F: \Omega-\bmod \rightarrow \bmod -\Gamma, \quad G: \bmod -\Gamma \rightarrow \Omega-\bmod .
$$

Theorem. Suppose $\Gamma$ and $\Omega$ are Morita-equivalent. Then

(1) $F$ and $G$ are both exact;

(2) $F G$ and $G F$ are both the identity;

(3) There is a one-to-one, inclusion-preserving correspondence between the twosided ideals in $\Gamma$ and the 2-sided ideals in $\Omega$.

COROLlaRY. If $\Gamma$ and $\Omega$ are Morita equivalent then

(1) mod- $\Gamma$ and $\Omega$-mod are isomorphic;

(2) $\mathrm{gl} \operatorname{dim} \Gamma=\mathrm{gl} \operatorname{dim} \Omega$;

(3) $\Gamma$ and $\Omega$ have the same number of maximal two-sided ideals. 
We return now to maximal orders.

THEOREM 6.6. Let $(R, \underline{m})$ be a regular local ring of dimension two. Let $\Lambda$ be a quasi-local $R$-order in $\Sigma$ such that $\mathrm{gl} \operatorname{dim} \Lambda<\infty$. Let $\Sigma^{\prime}$ be any central simple $K$ algebra Morita equivalent to $\Sigma$. Let $\Omega$ be any maximal $R$-order in $\Sigma^{\prime}$. Then $\Omega$ is Morita equivalent to some maximal $R$-order in $\Sigma$. Consequently $\Omega$ is quasi-local, $\mathrm{gl} \operatorname{dim} \Omega=2$, and all maximal $R$-orders in $\Sigma^{\prime}$ are isomorphic.

Proof. A slight modification of the proof of Theorem $6.5(\mathrm{~b})$ shows that if $\Sigma^{\prime}$ is Morita equivalent to $\Sigma$ and $\Omega$ is a maximal $R$-order in $\Sigma^{\prime}$, then there exists an $R$-reflexive left $\Omega$-module $E$ such that $\Gamma=\operatorname{End}_{\Omega}(E)$ is a maximal $R$-order in $\Sigma$ and $\Omega=\operatorname{End}_{\Gamma}(E)$. By Theorem $5.4 \mathrm{gldim} \Lambda=2, \Lambda$ is a maximal $R$-order in $\Sigma$, and all maximal $R$-orders in $\Sigma$ are isomorphic. So $\Gamma \simeq \Lambda$ and thus $\mathrm{gl} \operatorname{dim} \Gamma=2$ and $\Gamma$ is quasi-local. Since $E$ is $R$-reflexive and $\operatorname{gl} \operatorname{dim} R=2, E$ is $R$-free, so by Proposition $3.5 E$ is $\Gamma$-projective. Hence $\tau_{\Gamma}(E) \cdot E=E$. By Nakayama's Lemma, $\tau_{\Gamma}(E) \notin \operatorname{Rad} \Gamma$. But $\tau_{\Gamma}(E)$ is a two-sided ideal of $\Gamma$ and $\operatorname{Rad} \Gamma$ is the unique maximal two-sided ideal of $\Gamma$. Thus $\tau_{\Gamma}(E)=\Gamma$ and so $\Gamma$ is Morita equivalent to $\Omega$. Therefore $\mathrm{gl} \operatorname{dim} \Omega=2$ and $\Omega$ is quasi-local. Using Theorem 5.4 once again, we have that all maximal $R$-orders in $\Sigma^{\prime}$ are isomorphic.

CoRollary 6.7. Let $(R, \underline{m})$ be a complete regular local ring of dimension two. Let $\Delta$ be a central division algebra over $K$. Suppose $\Sigma$ is Morita equivalent to $\Delta$ (i.e., $\left.\Sigma=\operatorname{End}_{\Delta}(V)\right)$. A maximal $R$-order $\Lambda$ in $\Sigma$ is quasi-local and has global dimension two if and only if there exists a maximal $R$-order $\Omega$ in $\Delta$ which has global dimension two.

Proof. If $\Gamma$ is any $R$-order in $\Delta$, then by the completeness of $R$, any idempotent in $\Gamma / \operatorname{Rad} \Gamma$ can be lifted to an idempotent in $\Gamma$. However, the division ring $\Delta$ contains no proper idempotents. Hence $\Gamma / \operatorname{Rad} \Gamma$ has no proper idempotents, and since it is semisimple, it must be a division ring. Thus any $R$-order in $\Delta$ is local. The result now follows from Theorem 6.6.

The next theorem about Morita equivalence of quasi-local and local rings will enable us to give a generalization of Theorem 4.2 with which we shall conclude this paper.

THEOREM. 6.8. Let $\Gamma$ be a noetherian ring, $J=\operatorname{Rad} \Gamma$, and assume that $\Gamma$ is complete with respect to the J-adic topology. If $\Gamma$ is quasi-local, then there is a local ring $\Omega$ Morita equivalent to $\Gamma$, and $\Omega / \operatorname{Rad} \Omega$ is the division ring equivalent to $\Gamma / J$.

Proof. Let $I$ be a simple left ideal in $\Gamma / J$.

Then $I$ is generated by some idempotent $\xi$. Since $\Gamma$ is complete, $\xi$ can be lifted to an idempotent $e$ in $\Gamma$. Let $E=\Gamma e$. Then $E$ is $\Gamma$-projective. Let $\pi: \Gamma \rightarrow \Gamma / J$ be the natural projection $\pi(e)=\xi$ and $\pi(E)=I$. Clearly $J e \subset \operatorname{Ker}\left(\pi_{\mid E}\right)$. For the reverse 
inclusion, if $\gamma e \in J$ then $\gamma e=\lambda e \cdot e \in J e$. Thus $\operatorname{Ker}\left(\pi_{\mid E}\right)=J e$ and so $E / J E \approx I$. Therefore $E / J E$ is a simple $\Gamma / J$-module.

Now let $\Omega=\operatorname{End}_{\Gamma}(E)$ and $\Delta=\operatorname{End}_{\Gamma / J}(E / J E)$. Since $\Gamma$ is quasi-local and $E$ is $\Gamma$-projective, $\tau_{\Gamma}(E)=\Gamma$. Thus $\Omega$ is Morita equivalent to $\Gamma$. Hence $\Omega$ is quasi-local. By the Wedderburn theory, since $E / N E$ is simple, $\Delta$ is a division ring equivalent to $\Gamma / J$. We shall show that $\Omega / \operatorname{Rad} \Omega \approx \Delta$.

We have a ring homomorphism $\operatorname{End}_{\Gamma}(E) \rightarrow \operatorname{End}_{\Gamma / J}(E / J E)$ (i.e., $\Omega \rightarrow \Delta$ ) which is onto since $E$ is $\Gamma$-projective. The kernel is therefore a maximal two-sided ideal of $\Omega$. But $\Omega$ is quasi-local, so the kernel must be $\operatorname{Rad} \Omega$ and we $\operatorname{get} \Omega / \operatorname{Rad} \Omega \approx \Delta$. Thus $\Omega$ is local and $\Omega / \operatorname{Rad} \Omega$ is the division ring Morita equivalent to $\Gamma / J$.

THEOREM 6.9. Let $(R, \underline{m})$ be regular local of dimension $n$. Let $\Gamma \supset R$ be a quasilocal $R$-algebra, finitely generated as an $R$-module. Let $J=\operatorname{Rad} \Gamma$ and assume that $x \in \underline{m}-J^{2}$. Suppose $\mathrm{gl} \operatorname{dim} \Gamma<\infty$ (and thus equals $n$ ). Then $\mathrm{gl} \operatorname{dim} \Gamma / x \Gamma=n-1$.

Proof. All the hypotheses carry over to the completions $(\hat{R}, \underline{\hat{m}})$ and $(\hat{\Gamma}, \hat{J})$. [Note: the $J$-adic and the $\underline{m} \Gamma$-adic topologies on $\Gamma$ are the same.] Furthermore $\hat{\Gamma} / x \hat{\Gamma}=(\Gamma / x \Gamma)^{\wedge}$, so $\mathrm{gl} \operatorname{dim} \hat{\Gamma} / x \hat{\Gamma}=\mathrm{gl} \operatorname{dim} \Gamma / x \Gamma$. Thus it suffices to prove the theorem when $R$ is complete, which assumption we now make. Then by Theorem 6.8 $\Gamma$ is Morita equivalent to some local ring $\Omega$. Therefore $\mathrm{gl} \operatorname{dim} \Omega=\mathrm{gl} \operatorname{dim} \Gamma$. Suppose $x \in(\operatorname{Rad} \Omega)^{2}$. Then $x \Omega=\Omega x \subset(\operatorname{Rad} \Omega)^{2}$. Under the one-to-one correspondence of two-sided ideals in $\Omega$ and $\Gamma, x \Omega \leftrightarrow x \Gamma$ and $(\operatorname{Rad} \Omega)^{2} \leftrightarrow J^{2}$. Since the correspondence preserves inclusions we get $x \Gamma \subset J^{2}$, which is a contradiction. So $x \notin(\operatorname{Rad} \Omega)^{2}$. We may now apply Theorem 4.2 to the local ring $\Omega$ and conclude that $\mathrm{gl} \operatorname{dim} \Omega / x \Omega=n-1 . \Omega=\operatorname{End}_{\Gamma}(E)$ and $E$ is $\Gamma$-projective. Since $\Gamma$ is $R$-free (Corollary 2.17), $E$ is $R$-free and thus $x$ is regular on $E$. This together with the $\Gamma$ projectivity of $E$ implies that $\Omega / x \Omega \approx E \ln _{\Gamma / x \Gamma}(E / x E)$. Also, $E / x E$ is $\Gamma / x \Gamma$-projective and since $\tau_{\Gamma}(E)=\Gamma$ it follows that $\tau_{\Gamma / x \Gamma}(E / x E)=\Gamma / x \Gamma$. So $\Omega / x \Omega$ is Morita equivalent to $\Gamma / x \Gamma$ and therefore $\operatorname{gl} \operatorname{dim} \Gamma / x \Gamma=\operatorname{gl} \operatorname{dim} \Omega / x \Omega=n-1$.

\section{BIBLIOGRAPHY}

1. M. Auslander, On the dimension of modules and algebras. III, Nagoya Math. J. 9 (1955), 67-77.

2. —, Remarks on a theorem of Bourbaki, Nagoya Math. J. 27 (1966), 361-369.

3. M. Auslander and D. Buchsbaum, Homological dimension in local rings, Trans. Amer. Math. Soc. 85 (1957), 390-405.

4. M. Auslander and O. Goldman, Maximal orders, Trans. Amer. Math. Soc. 97 (1960), 1-24.

5. - The Brauer group of a commutative ring, Trans. Amer. Math. Soc. 97 (1960), 367-409.

6. H. Bass, Injective dimension in noetherian rings, Trans. Amer. Math. Soc. 102 (1962), 18-29.

7. N. Bourbaki, Algèbre commutative, Chapitre 7, Diviseurs, Actualités Sci. Indust. No. 1314, Hermann, Paris, 1965. 
8. H. Cartan and S. Eilenberg, Homological algebra, Princeton Univ. Press, Princeton, N. J., 1956.

9. S. Eilenberg and T. Nakayama, On the dimension of modules and algebras. II, Nagoya Math. J. 9 (1955), 1-16.

10. E. Matlis, Applications of duality, Proc. Amer. Math. Soc. 10 (1959), 659-662.

11. L. Silver, Noncommutative localizations, J. Algebra 7 (1967), 44-76.

\section{HaRvard UNIVERSITY,}

Cambridge, Massachusetts 